\title{
FINITE ELEMENT ERROR ESTIMATES ON THE BOUNDARY WITH APPLICATION TO OPTIMAL CONTROL
}

\author{
THOMAS APEL, JOHANNES PFEFFERER, AND ARND RÖSCH
}

\begin{abstract}
This paper is concerned with the discretization of linear elliptic partial differential equations with Neumann boundary condition in polygonal domains. The focus is on the derivation of error estimates in the $L^{2}$-norm on the boundary for linear finite elements. Whereas common techniques yield only suboptimal results, a new approach in this context is presented which allows for quasi-optimal ones, i.e., for domains with interior angles smaller than $2 \pi / 3$ a convergence order two (up to a logarithmic factor) can be achieved using quasi-uniform meshes. In the presence of internal angles greater than $2 \pi / 3$ which reduce the convergence rates on quasi-uniform meshes, graded meshes are used to maintain the quasi-optimal error bounds.

This result is applied to linear-quadratic Neumann boundary control problems with pointwise inequality constraints on the control. The approximations of the control are piecewise constant. The state and the adjoint state are discretized by piecewise linear finite elements. In a postprocessing step approximations of the continuous optimal control are constructed which possess superconvergence properties. Based on the improved error estimates on the boundary and optimal regularity in weighted Sobolev spaces almost second order convergence is proven for the approximations of the continuous optimal control problem. Mesh grading techniques are again used for domains with interior angles greater than $2 \pi / 3$. A certain regularity of the active set is assumed.
\end{abstract}

\section{INTRODUCTION}

Let $\Omega$ be a bounded, two-dimensional, polygonal domain with Lipschitz boundary $\Gamma$ and $m$ corner points $x^{(j)}, j=1, \ldots, m$, counting counterclockwise. In particular, $\Gamma_{j}$ denotes the side on the boundary $\Gamma$ which connects the corners $x^{(j)}$ and $x^{(j+1)}$; note that $x^{(m+1)}:=x^{(1)}$. The angle between $\Gamma_{j-1}$ and $\Gamma_{j}$ is denoted by $\omega_{j}$ with the obvious modification for $\omega_{1}$. In the first part of this paper, we will discretize the linear elliptic partial differential equation

$$
\begin{aligned}
-\Delta y+y=f & \text { in } \Omega, \\
\partial_{n} y=g & \text { on } \Gamma_{j}, \quad j=1, \ldots, m,
\end{aligned}
$$

with piecewise linear and continuous ansatz functions and focus on finite element error estimates in the $L^{2}$-norm on the boundary. Common approaches for this use the trace theorem or the Nitsche method in $L^{2}(\Gamma)$. This yields an error bound

Received by the editor November 19, 2012 and, in revised form, May 16, 2013.

2010 Mathematics Subject Classification. Primary 65N30, 49M25, 65N50, 65N15.

Key words and phrases. Linear-quadratic Neumann boundary control problem, control constraints, corner singularities, weighted Sobolev spaces, finite element method, error estimates, boundary estimates, quasi-uniform meshes, graded meshes, postprocessing, superconvergence. 
of $c h^{3 / 2}$. This estimate is sharp in the case of $H^{2}(\Omega)$-regularity of the solution. For example, such limited regularity can be caused by the presence of corner singularities or by right-hand sides $f$ and $g$ in $L^{2}(\Omega)$ and $H^{1 / 2}(\Gamma)$, respectively. For more regular solutions in $W^{2, p}(\Omega)$ with $p>2$ numerical examples indicate that the convergence order is better; cf. [1,20]. This can be explained by first using the embedding $L^{p}(\Gamma) \hookrightarrow L^{2}(\Gamma)$ and then applying the trace theorem or the Nitsche method in $L^{p}(\Gamma)$. Similar techniques are used in [20]. By this one obtains an error bound of $c h^{2-1 / p}$. Accordingly for problem (1.1) a convergence order close to 2 can only be expected if $\omega_{j}<\pi / 2$ for $j=1, \ldots, m$, since the corner singularities admit in general only a solution in $W^{2, \infty}(\Omega)$ for such domains. In the present work we will show that the estimates can even be improved assuming Hölder continuous right-hand sides. Based on regularity results in weighted Sobolev spaces, techniques of [2, 29, 30] and local finite element error estimates as described in [9, 28, 32, for a quasi-optimal error bound of $c h^{2}|\ln h|^{1+\delta}$ with some $\delta \in[0,1 / 2]$ will be obtained for domains with interior angles smaller than $\pi /(2-\delta)$. Hence, in domains with interior angles smaller than $2 \pi / 3$ the error is definitely bounded by $c h^{2}|\ln h|^{3 / 2}$. These estimates hold still for quasi-uniform meshes. For domains with larger interior angles than $\pi /(2-\delta)$ mesh grading techniques will be applied to get the same result. In that case the mesh grading parameter $\mu_{j}$, that determines the strength of the grading around the corner $x^{(j)}$, has to be chosen smaller than $\left(\delta+\pi / \omega_{j}\right) / 2$; see Section 3 for details.

Error estimates of that kind are, for example, required for the numerical analysis of Neumann boundary control problems. The second part of this paper is dedicated to this topic. We will consider the optimal control problem

$$
\begin{aligned}
J(\bar{u}) & =\min _{u \in U_{a d}} J(u), \\
J(u) & :=F(S u, u), \\
F(y, u) & :=\frac{1}{2}\left\|y-y_{d}\right\|_{L^{2}(\Omega)}^{2}+\frac{\nu}{2}\|u\|_{L^{2}(\Gamma)}^{2},
\end{aligned}
$$

where the associated state $y=S u$ to the control $u$ is the weak solution of

$$
\begin{aligned}
-\Delta y+y=0 & \text { in } \Omega, \\
\partial_{n} y=u & \text { on } \Gamma_{j}, \quad j=1, \ldots, m,
\end{aligned}
$$

the desired state $y_{d}$ belongs to the Hölder space $C^{0, \sigma}(\bar{\Omega})$ with some $\sigma \in(0,1)$ and the control variable is constrained by

$$
a \leq u(x) \leq b \text { for a.a. } x \in \Gamma .
$$

We will focus on the full discretization of problem (1.2) combined with a postprocessing procedure, i.e., the state and the adjoint state are discretized by linear finite elements and the control is approximated by piecewise constant functions on the boundary. Afterwards, in a postprocessing step, piecewise linear and globally continuous controls are constructed, which possess superconvergence properties. This postprocessing approach is well known for linear optimal control problems; see [3, 24] for distributed controls and [1,20] for controls located at the Neumann boundary. It is extended to semilinear control problems in [26]. Also, different approaches for problem (1.2) have been discussed in the literature. The numerical analysis for the full discretization itself has been accomplished in [7]. In [6] the control is not discretized by piecewise constant functions but by piecewise linear and 


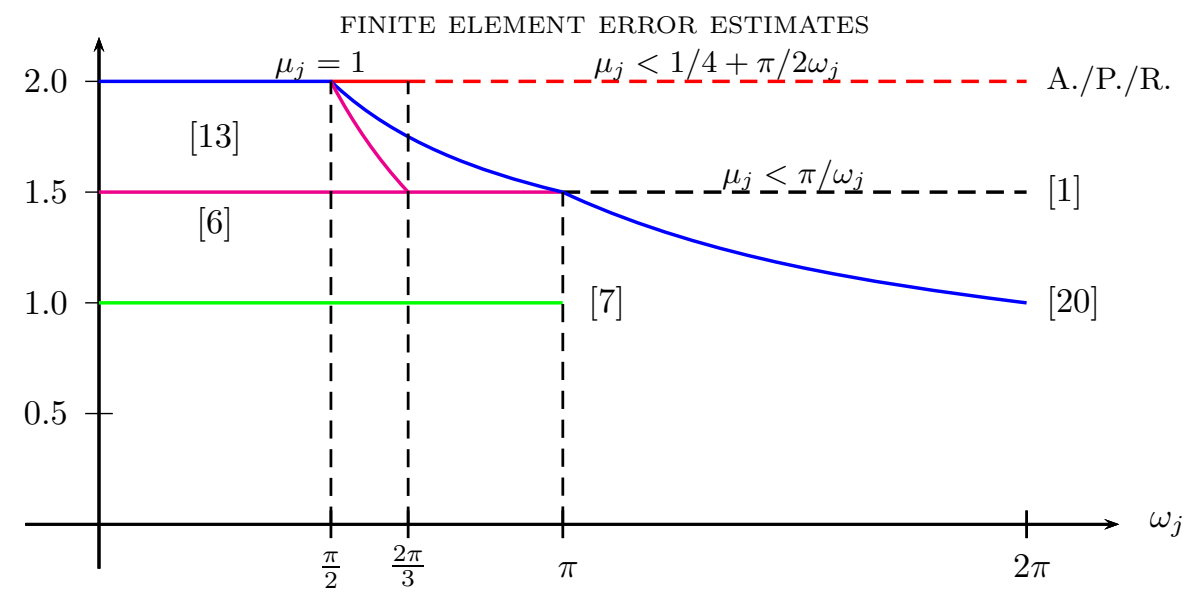

Figure 1. Convergence rates of the control in $L^{2}(\Gamma)$ depending on the interior angles $\omega_{j}$. Solid lines: quasi-uniform meshes. Dashed lines: graded meshes.

globally continuous functions. These two works attack semilinear elliptic problems. The variational discretization concept, originally suggested in [12] for distributed control problems, has been transferred to Neumann boundary control problems in [6] for semilinear and in [1,13, 20, for linear elliptic equations. This approach only discretizes the state and adjoint state by linear finite elements but not the control. In Figure 1 the obtained convergence rates of the control in $L^{2}(\Gamma)$ for the different approaches are illustrated depending on the interior angles of the domain. In [7 a convergence order of 1 is obtained in convex domains for the full discretization of semilinear elliptic problems, where the control is discretized piecewise constantly. Approximating the control with piecewise linear and continuous functions implies, in convex domains, superlinear convergence and a convergence rate of $3 / 2$ under an additional assumption on the control; see [6]. Furthermore, in [6] an error bound in $L^{2}(\Gamma)$ of $c h^{3 / 2-\epsilon}$ with some arbitrary $\epsilon>0$ is established in convex domains for the concept of variational discretizations. This approach admits, for linear problems in convex domains, a convergence rate in $L^{2}(\Gamma)$ of $3 / 2$ and an error bound of $c h^{2-2 / p}|\log h|$ in $L^{\infty}(\Gamma)\left(\hookrightarrow L^{2}(\Gamma)\right)$ with $2<p<\min _{j}\left(2 \omega_{j} /\left(2 \omega_{j}-\pi\right)\right)$; see [13]. An improved estimate for the concept of variational discretizations and the postprocessing approach can be found in [20]. There, the authors proved for convex domains an approximation rate of $2-1 / p$ with the parameter $p$ as before and a rate of $\min _{j}\left(1 / 2+\pi / \omega_{j}\right)$ for nonconvex domains. They further establish better estimates using higher order finite elements for the discretization of the state and adjoint state (not contained in Figure 1). All the results, stated so far, have in common, that the convergence rates are lower than $3 / 2$ in nonconvex domains. In [1] we have already proved that graded meshes with grading parameters $\mu_{j}<\pi / \omega_{j}$ can be used to maintain a convergence order of $3 / 2$ in nonconvex domains. Using the quasi-optimal error estimates on the boundary of the first part and optimal regularity of the solution in weighted Sobolev spaces we will show in the present work under a structural assumption on the active set, that the error is bounded by $c h^{2}|\ln h|^{3 / 2}$ using quasi-uniform meshes even if $\omega_{j}<2 \pi / 3$. For larger interior angles we will prove that graded meshes with $\mu_{j}<1 / 4+\pi / 2 \omega_{j}$ imply the same 
convergence rate. For further literature concerning optimal control problems and its discretization we refer to [1, 14, 20, 31] and the references therein.

The paper is organized as follows: In the next section we introduce weighted spaces and some of its properties, which are used for the analysis in the subsequent sections. Furthermore, we state results about weighted $W^{2, \infty}$-regularity of the solution of problem (1.1). The proof of these results is based on regularity results in weighted Hölder spaces and is postponed to Section 8. Section 3 is concerned with the discretization of problem (1.1) by linear finite elements using quasi-uniform and graded meshes. This section includes the quasi-optimal finite element error estimates in the domain and our first main result, the quasi-optimal finite element error estimate on the boundary. In Section 4 we discuss the necessary and sufficient optimality condition of problem (1.2). As a key point for the subsequent numerical analysis we also prove optimal regularity in weighted Sobolev spaces for the solution of the optimal control problem. The discrete counterpart of the continuous optimal control problem (1.2) and its necessary and sufficient optimality condition is stated in Section 5. In Section 6 we prove interpolation error estimates on the boundary. These are special compared to the interpolation error estimates of Section 3, since the techniques used in Section 3 would only yield suboptimal results. Finally, quasioptimal error estimates for the postprocessing approach are contained in Section 7 We emphasize, that the optimal control and the desired state are separated from the constants in all estimates.

In the sequel $c$ denotes a generic constant, which is always independent of the discretization parameter.

\section{Regularity in Weighted Sobolev spaces}

The regularity of the solution of the elliptic boundary value problem (1.1) is in general limited due to the corners of the domain even if the data $f$ and $g$ are smooth. If one uses classical Sobolev-Slobodetskij spaces $W^{s, p}(\Omega)$ to describe the regularity, then this limitation is given by a dependence of the parameters $s$ and $p$ on the interior angles of the domain; compare e.g. [8, 11]. Another possibility to state the regularity uses weighted Sobolev spaces, which better incorporates the singular behavior coming from the corners. To this end we introduce the circular sectors $\Omega_{R_{j}}$ and $\Omega_{R_{j} / 64}$ which are centered at the corners $x^{(j)}$ and possess the opening angles $\omega_{j}$ and the radii $R_{j}$ and $R_{j} / 64$, respectively. The radii $R_{j}$ can be chosen arbitrarily with the only restriction that the circular sectors $\Omega_{R_{j}}$ do not overlap. We denote by $r_{j}$ and $\varphi_{j}$ the polar coordinates located at the point $x^{(j)}$. The sides of the circular sectors $\Omega_{R_{j}}$, which coincide with the boundary $\Gamma$ locally, are defined by $\Gamma_{j}^{+}\left(\varphi_{j}=\omega_{j}\right)$ and $\Gamma_{j}^{-}\left(\varphi_{j}=0\right)$. We set $\Gamma_{j}^{ \pm}=\Gamma_{j}^{+} \cup \Gamma_{j}^{-}$. In the following the closure of some set $G$ will be denoted either by $\bar{G}$ or by $\operatorname{cl}(G)$. We set

$$
\Omega^{0}=\Omega \backslash \bigcup_{j=1}^{m} \Omega_{R_{j} / 64} \quad \text { and } \quad \Gamma^{0}=\Gamma \cap \bar{\Omega}^{0} .
$$

We define for $k \in \mathbb{N}_{0}, p \in[1, \infty]$ and $\vec{\beta}=\left(\beta_{1}, \ldots, \beta_{m}\right)^{T} \in \mathbb{R}^{m}$ the weighted Sobolev spaces $W_{\vec{\beta}}^{k, p}(\Omega)$ as the set of all functions on $\Omega$ with finite norm

$$
\|v\|_{W_{\vec{\beta}}^{k, p}(\Omega)} \sim\|v\|_{W^{k, p}\left(\Omega^{0}\right)}+\sum_{j=1}^{m}\|v\|_{W_{\beta_{j}}^{k, p}\left(\Omega_{R_{j}}\right)},
$$


where the Sobolev spaces $W^{k, p}(\Omega)\left(=H^{k}(\Omega)\right.$ for $\left.p=2\right)$ are defined as usual and the weighted part in the norm is defined by

$$
\begin{aligned}
\|v\|_{W_{\beta_{j}}^{k, p}\left(\Omega_{R_{j}}\right)} & =\left(\sum_{|\alpha| \leq k}\left\|r_{j}^{\beta_{j}} D^{\alpha} v\right\|_{L^{p}\left(\Omega_{R_{j}}\right)}^{p}\right)^{1 / p} \text { if } 1 \leq p<\infty, \\
\|v\|_{W_{\beta_{j}}^{k, \infty}\left(\Omega_{R_{j}}\right)} & =\sum_{|\alpha| \leq k}\left\|r_{j}^{\beta_{j}} D^{\alpha} v\right\|_{L^{\infty}\left(\Omega_{R_{j}}\right)}
\end{aligned}
$$

using standard multi-index notation. We denote by $\mathcal{C}$ the set of all corner points. The corresponding trace spaces $W_{\vec{\beta}}^{k-1 / p, p}(\Gamma)(k \geq 1)$ are equipped with the norm

$$
\|v\|_{W_{\vec{\beta}}^{k-1 / p, p}(\Gamma)}=\inf \left\{\|u\|_{W_{\vec{\beta}}^{k, p}(\Omega)}: u \in W_{\vec{\beta}}^{k, p}(\Omega), u_{\mid \Gamma \backslash \mathcal{C}}=v\right\} .
$$

Furthermore, we define the space $W_{\vec{\beta}}^{k, p}(\Gamma)$ for $k \in \mathbb{N}_{0}, p \in[1, \infty]$ and $\vec{\beta}=\left(\beta_{1}, \ldots, \beta_{m}\right)^{T}$ $\in \mathbb{R}^{m}$ as the space of all functions such that

$$
\|v\|_{W_{\vec{\beta}}^{k, p}(\Gamma)} \sim\|v\|_{W^{k, p}\left(\Gamma^{0}\right)}+\sum_{j=1}^{m}\|v\|_{W_{\beta_{j}}^{k, p}\left(\Gamma_{j}^{ \pm}\right)},
$$

is finite, where

$$
\begin{aligned}
\|v\|_{W_{\beta_{j}}^{k, p}\left(\Gamma_{j}^{ \pm}\right)} & =\left(\sum_{|\alpha| \leq k}\left(\left\|r_{j}^{\beta_{j}} \partial_{t}^{\alpha} v\right\|_{L^{p}\left(\Gamma_{j}^{+}\right)}^{p}+\left\|r_{j}^{\beta_{j}} \partial_{t}^{\alpha} v\right\|_{L^{p}\left(\Gamma_{j}^{-}\right)}^{p}\right)\right)^{1 / p} \text { if } 1 \leq p<\infty, \\
\|v\|_{W_{\beta_{j}}^{k, \infty}\left(\Gamma_{j}^{ \pm}\right)} & =\sum_{|\alpha| \leq k}\left(\left\|r_{j}^{\beta_{j}} \partial_{t}^{\alpha} v\right\|_{L^{\infty}\left(\Gamma_{j}^{+}\right)}+\left\|r_{j}^{\beta_{j}} \partial_{t}^{\alpha} v\right\|_{L^{\infty}\left(\Gamma_{j}^{-}\right)}\right) .
\end{aligned}
$$

Note, that $\partial_{t} v$ denotes the tangential derivative of $v$. For the numerical analysis it is useful to introduce the seminorms

$$
|\cdot|_{W_{\vec{\beta}}^{k, p}(\Omega)} \text { and }|\cdot|_{W_{\vec{\beta}}^{k, p}(\Gamma)},
$$

which are defined in analogy to the classical Sobolev seminorms. In Lemmas 2.1 and 2.2 we state selected properties of the weighted Sobolev spaces $W_{\vec{\beta}}^{k, p}(\Omega)$ and $W_{\vec{\beta}}^{k, p}(\Gamma)$ where we will use the uniform notation $W_{\vec{\beta}}^{k, p}(\mathcal{G})$. The dimension of $\mathcal{G}$ is $n=2$ when $\mathcal{G}=\Omega$ (or part of it) and $n=1$ when $\mathcal{G}=\Gamma$ (or part of it).

Lemma 2.1. Let $l$ and $k$ be nonnegative integers. Then the following assertions hold:

(1) Let $\beta_{j}^{\prime}>-n / p$ and $\beta_{j}-\beta_{j}^{\prime} \leq k$ for $j=1, \ldots, m$ and $1 \leq p<\infty$. Then the continuous embedding $W_{\vec{\beta}}^{l+k, p}(\mathcal{G}) \hookrightarrow W_{\vec{\beta}^{\prime}}^{l, p}(\mathcal{G})$ holds.

(2) Let $n / q-n / p>\beta_{j}-\beta_{j}^{\prime}$ for $j=1, \ldots, m$ and $1 \leq q<p \leq \infty$. Then the continuous embedding $W_{\vec{\beta}}^{l, p}(\mathcal{G}) \hookrightarrow W_{\vec{\beta}^{\prime}}^{l, q}(\mathcal{G})$ is valid.

(3) Let $\beta_{j}^{\prime}>-n / p$ and $\beta_{j}-\beta_{j}^{\prime}<1$ for $j=1, \ldots, m$ and $1 \leq p<\infty$. Then the compact embedding $W_{\vec{\beta}}^{l+1, p}(\mathcal{G}) \stackrel{c}{\hookrightarrow} W_{\vec{\beta}^{\prime}}^{l, p}(\mathcal{G})$ holds. 
Proof. 1. Let $\gamma_{j}:=\beta_{j}^{\prime}+k$ and $\vec{\gamma}=\left(\gamma_{1}, \ldots, \gamma_{m}\right)$. By Hardy's inequality applied $k$-times and embeddings in the usual Sobolev spaces one obtains for $\beta_{j}^{\prime}>-n / p$ that

$$
W_{\vec{\gamma}}^{l+k, p}(\mathcal{G}) \hookrightarrow W_{\vec{\beta}^{\prime}}^{l, p}(\mathcal{G}) ;
$$

cf. Lemma 7.1.5 in [16] for the two-dimensional case with $p=2$, and [18, (0.35)] for general $p$ but slightly different notation. Now, the first assertion follows immediately since

$$
W_{\vec{\beta}}^{l+k, p}(\mathcal{G}) \hookrightarrow W_{\vec{\gamma}}^{l+k, p}(\mathcal{G})
$$

for $\beta_{j} \leq \gamma_{j}$ which is equivalent to $\beta_{j}-\beta_{j}^{\prime} \leq k$.

2. This is a consequence of the Hölder inequality.

3. For three space dimensions this is proven in Lemma 8.1.2 in [21]. In one and two space dimensions it can be proven analogously using the continuous embedding of 1 .

Lemma 2.2. Let $q \in[1, \infty),-n / q<\beta_{j}<n-n / q+1$ for $j=1, \ldots, m, k \geq 0$ and $v \in W_{\beta}^{k+1, q}(\mathcal{G})$. Then the norm equivalence

$$
\|v\|_{W_{\vec{\beta}}^{k+1, q}(\mathcal{G})} \sim|v|_{W_{\vec{\beta}}^{k+1, q}(\mathcal{G})}+\sum_{|\delta| \leq k}\left|\int_{\mathcal{G}} D^{\delta} v d x\right|
$$

is valid.

Proof. This assertion has already been proven in Lemma 2.2 of [4], where the authors assume that $1-2 / q<\beta_{j} \leq 1$. Let $\overrightarrow{1}=(1, \ldots, 1) \in \mathbb{R}^{m}$. Using Lemma 2.1 parts 2,1 , and 3 , respectively, one has

$$
W_{\vec{\beta}}^{k+1, q}(\mathcal{G}) \hookrightarrow W_{\overrightarrow{1}}^{k+1,1}(\mathcal{G}) \hookrightarrow W^{k, 1}(\mathcal{G}) \text { and } W_{\vec{\beta}}^{k+1, q}(\mathcal{G}) \stackrel{c}{\hookrightarrow} W_{\vec{\beta}}^{k, q}(\mathcal{G})
$$

for $-n / q<\beta_{j}<n-n / q+1$. These two embeddings are essential to prove the norm equivalence (2.1). In fact, tracing through the proof of Lemma 2.2 in [4] reveals that the condition $1-2 / q<\beta_{j} \leq 1$ can simply be replaced by $-n / q<\beta_{j}<n-n / q+1$ by means of (2.2).

As usual, the space $C^{k}(\bar{\Omega})$ denotes the set of all functions on $\Omega$ with bounded and uniformly continuous derivatives up to order $k$. The norm in $C^{k}(\bar{\Omega})$ is defined by

$$
\|v\|_{C^{k}(\bar{\Omega})}=\sum_{|\alpha| \leq k} \sup _{x \in \Omega}\left|D_{x}^{\alpha} v(x)\right| .
$$

Functions belonging to the Hölder space $C^{k, \sigma}(\bar{\Omega})$ additionally possess bounded derivatives of order $k$ which are Hölder continuous with exponent $\sigma \in(0,1)$. The norm in the Hölder space $C^{k, \sigma}(\bar{\Omega})$ is given by

$$
\|v\|_{C^{k, \sigma}(\bar{\Omega})}=\|v\|_{C^{k}(\bar{\Omega})}+\sum_{|\alpha|=k} \sup _{x, y \in \Omega} \frac{\left|D_{x}^{\alpha} v(x)-D_{y}^{\alpha} v(y)\right|}{|x-y|^{\sigma}} .
$$

Now we have everything at hand to state the regularity results. Let $f$ and $g$ be elements of the dual spaces of $H^{1}(\Omega)$ and $H^{1 / 2}(\Gamma)$, respectively. Then, the generalized solution of (1.1) is the unique element $y \in H^{1}(\Omega)$ that satisfies

$$
a(y, v)=(f, v)_{L^{2}(\Omega)}+(g, v)_{L^{2}(\Gamma)} \quad \forall v \in H^{1}(\Omega),
$$


where $a: H^{1}(\Omega) \times H^{1}(\Omega) \rightarrow \mathbb{R}$ is the bilinear form

$$
a(y, v)=\int_{\Omega}(\nabla y \cdot \nabla v+y v) \mathrm{d} x .
$$

The regularity of the solution of problem (1.1) is described in the following two lemmata. The proofs are postponed to Section 8 , page 64f. A survey of regularity results of more general situations is contained in [19].

Lemma 2.3. Let $\lambda_{j}=\pi / \omega_{j}$ and let $\beta_{j}$ satisfy the condition

$$
1>\beta_{j}>\max \left(0,1-\lambda_{j}\right) \quad \text { or } \beta_{j}=0 \text { and } 1-\lambda_{j}<0
$$

for $j=1, \ldots, m$. Furthermore, let $f \in W_{\vec{\beta}}^{0,2}(\Omega)$ and $g \in W_{\vec{\beta}}^{1 / 2,2}(\Gamma)$. Then problem (1.1) has a unique generalized solution $y \in W_{\vec{\beta}}^{2,2}(\Omega)$ and the a priori estimate

$$
\|y\|_{W_{\vec{\beta}}^{2,2}(\Omega)} \leq c\left(\|f\|_{W_{\vec{\beta}}^{0,2}(\Omega)}+\|g\|_{W_{\vec{\beta}}^{1 / 2,2}(\Gamma)}\right)
$$

is valid.

Lemma 2.4. Let $\lambda_{j}=\pi / \omega_{j}$ and let $\gamma_{j}$ satisfy the condition

$$
2>\gamma_{j}>\max \left(0,2-\lambda_{j}\right) \quad \text { or } \quad \gamma_{j}=0 \text { and } 2-\lambda_{j}<0
$$

for $j=1, \ldots, m$. Moreover, let $f \in C^{0, \sigma}(\bar{\Omega})$ with $\sigma \in(0,1)$ and $g \equiv 0$. Then the unique generalized solution y of problem (1.1) fulfills the a priori estimate

$\|y\|_{W_{\vec{\gamma}}^{2, \infty}(\Omega)}+\|y\|_{W_{\vec{\gamma}}^{2, \infty}(\Gamma)} \leq c\left(\|y\|_{C^{2}\left(\bar{\Omega}^{0}\right)}+\sum_{j=1}^{m} \sum_{|\alpha| \leq 2}\left\|r_{j}^{\gamma_{j}} D^{\alpha} y\right\|_{C^{0}\left(\bar{\Omega}_{R_{j}}\right)}\right) \leq c\|f\|_{C^{0, \sigma}(\bar{\Omega})}$.

\section{Finite ELEMENT ERROR ESTimates ON the BOUNDARY}

We will now discretize the boundary value problem (1.1) by a finite element method. For this purpose we introduce a family of graded triangulations $\left\{\mathcal{T}_{h}\right\}$ of the domain $\Omega$ where $h$ denotes the global mesh parameter (cf. Definition 4.4.13 in [5]). We assume $h \leq h_{0}<1$. Note that a segmentation $\mathcal{E}_{h}$ of the boundary is naturally introduced by the triangulation $\mathcal{T}_{h}$. We denote by $\mu_{j} \in(0,1], j=1, \ldots, m$, the mesh grading parameters which are collected in the vector $\vec{\mu}$. The distance of the triangle $T$ to the corner $x^{(j)}$ is defined by $r_{T, j}:=\inf _{x \in T}\left|x-x^{(j)}\right|$. We assume that the element size $h_{T}:=\operatorname{diam} T$ satisfies

$$
\begin{aligned}
c_{1} h^{1 / \mu_{j}} & \leq h_{T} \leq c_{2} h^{1 / \mu_{j}} & & \text { for } r_{T, j}=0, \\
c_{1} h r_{T, j}^{1-\mu_{j}} & \leq h_{T} \leq c_{2} h r_{T, j}^{1-\mu_{j}} & & \text { for } 0<r_{T, j} \leq R_{j}, \\
c_{1} h & \leq h_{T} \leq c_{2} h & & \text { for } r_{T, j}>R_{j}
\end{aligned}
$$

for $j=1, \ldots, m$ and the radii $R_{j}$ which we have defined in Section 2 Next, we introduce the space $V_{h}$ as the space of all piecewise linear and globally continuous functions in $\bar{\Omega}$,

$$
V_{h}:=\left\{y_{h} \in C(\bar{\Omega}):\left.y_{h}\right|_{T} \in \mathcal{P}_{1}(T) \forall T \in \mathcal{T}_{h}\right\},
$$

where $\mathcal{P}_{1}(T)$ denotes the space of polynomials of degree less than or equal to one on $T$.

The discrete solution is the unique element $y_{h} \in V_{h} \subset H^{1}(\Omega)$ that satisfies

$$
a\left(y_{h}, v_{h}\right)=\left(f, v_{h}\right)_{L^{2}(\Omega)}+\left(g, v_{h}\right)_{L^{2}(\Gamma)} \quad \forall v_{h} \in V_{h} .
$$


We set $\vec{\lambda}=\left(\lambda_{1}, \ldots, \lambda_{m}\right)^{T}=\left(\pi / \omega_{1}, \ldots, \pi / \omega_{m}\right)^{T}$ and $\vec{a}=(a, \ldots, a)^{T} \in \mathbb{R}^{m}$ for any real number $a$, e.g., $\overrightarrow{1}=(1, \ldots, 1)^{T} \in \mathbb{R}^{m}$. Furthermore, all inequalities in the sequel containing vectorial parameters must be understood component-bycomponent. We proved in [1] the following estimates for the discretization error in the domain.

Theorem 3.1. Let $y$ and $y_{h}$ be the solutions of (2.3) and (3.2), respectively. The discretization error can be estimated by

$\left\|y-y_{h}\right\|_{L^{2}(\Omega)}+h\left\|y-y_{h}\right\|_{W^{1,2}(\Omega)} \leq c h^{2}\|y\|_{W_{\vec{\beta}}^{2,2}(\Omega)} \leq c h^{2}\left(\|f\|_{W_{\vec{\beta}}^{0,2}(\Omega)}+\|g\|_{W_{\vec{\beta}}^{1 / 2,2}(\Gamma)}\right)$,

provided that $\overrightarrow{1}-\vec{\lambda}<\vec{\beta} \leq \overrightarrow{1}-\vec{\mu}, \vec{\beta} \geq \overrightarrow{0}, f \in W_{\vec{\beta}}^{0,2}(\Omega)$ and $g \in W_{\vec{\beta}}^{1 / 2,2}(\Gamma)$.

The main new result of this section is the following finite element error estimate on the boundary.

Theorem 3.2. Let $y$ and $y_{h}$ be the solution of (2.3) and (3.2), respectively, and $g \equiv 0$. The finite element error on the boundary admits for some arbitrary $\delta \in[0,1 / 2]$ the estimate

$$
\left\|y-y_{h}\right\|_{L^{2}(\Gamma)} \leq c h^{2}|\ln h|^{1+\delta}\|f\|_{C^{0, \sigma}(\bar{\Omega})}
$$

provided that $\vec{\mu}<\vec{\delta} / 2+\vec{\lambda} / 2, \vec{\mu} \in(\delta / 2,1]^{m}$ and $f \in C^{0, \sigma}(\bar{\Omega}), \sigma \in(0,1)$.

Before we prove this theorem we add some discussion.

Remark 3.3. To get optimal discretization error estimates in the domain one only needs a graded mesh with grading parameters $\vec{\mu}<\vec{\lambda}$ if the largest interior angle in the domain is larger than $\pi$. However, the stronger condition $\vec{\mu}<\overrightarrow{1} / 4+\vec{\lambda} / 2$ is required to guarantee a finite element error estimate on the boundary of order $O\left(h^{2}|\ln h|^{3 / 2}\right)$. Numerical examples also indicate that this condition is sharp; cf. [1. Mesh grading with the stronger refinement condition is indeed necessary in domains which have interior angles greater than $2 \pi / 3$.

Remark 3.4. In Theorem 3.2 a homogeneous boundary datum $g$ is assumed. However, the assertion can easily be generalized to the inhomogeneous case. Then one only needs regularity results as in Lemma 2.4 which incorporate an inhomogeneous boundary datum $g$.

Remark 3.5. Optimal finite element error estimates in the $L^{2}$-norm on a strip at the boundary with width $h$ are closely related to the error estimate of Theorem 3.2 In [23] the authors prove an optimal estimate on a strip for the Dirichlet problem in convex polygonal and polyhedral domains using quasi-uniform meshes. Whereas the general approach in 23 as well as in the present work relies on local finite element error estimates as described in [9, 32], the regularity theory used for the numerical analysis differs fundamentally. In [23] weighted and anisotropic spaces are used, which employ the distance to the boundary. In contrast, our analysis is based on weighted spaces with respect to the corners, which allow the usage of graded meshes with local grading parameters $\mu_{j}$ depending on the interior angles $\omega_{j}$ of each particular corner.

The remainder of this section is devoted to the proof of Theorem 3.2 The reader who is not interested in the proving techniques may jump directly to Section 4 (application to an optimal control problem), page 52 

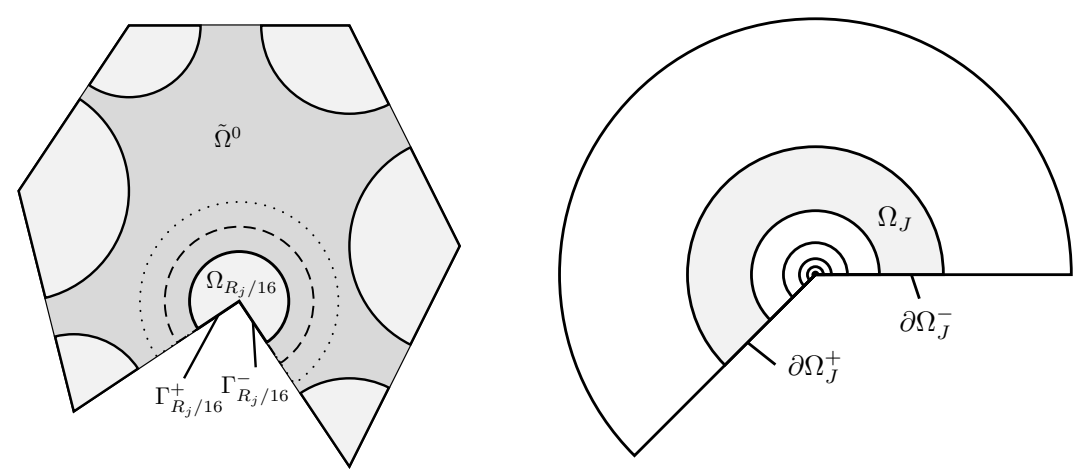

Figure 2. Partition of $\Omega$ with subdomains $\Omega_{R_{j} / 16}$ (left) and partition of $\Omega_{R}$ with subdomains $\Omega_{J}$ (right)

In Section 2 we have already introduced circular sectors $\Omega_{R_{j}}$ and $\Omega_{R_{j} / 64}$ which are centered at the corners $x^{(j)}$ and possess the radii $R_{j}$ and $R_{j} / 64$; compare also the mesh condition (3.1). Next, we introduce the circular sectors $\Omega_{R_{j} / 2}, \Omega_{R_{j} / 4}, \Omega_{R_{j} / 8}$, $\Omega_{R_{j} / 16}$ and $\Omega_{R_{j} / 32}$ with the radii $R_{j} / 2, R_{j} / 4, R_{j} / 8, R_{j} / 16$ and $R_{j} / 32$, respectively. The sides of the circular sectors $\Omega_{R_{j} / 16}$ which coincide with the boundary $\Gamma$ are denoted by $\Gamma_{R_{j} / 16}^{+}\left(\varphi_{j}=\omega_{j}\right)$ and $\Gamma_{R_{j} / 16}^{-}\left(\varphi_{j}=0\right)$. The union of both is denoted by $\Gamma_{R_{j} / 16}^{ \pm}$. Furthermore, we define $\tilde{\Omega}^{0}=\Omega \backslash \bigcup_{j=1}^{m} \Omega_{R_{j} / 16}, \tilde{\Gamma}^{0}=\Gamma \cap \operatorname{cl}\left(\tilde{\Omega}^{0}\right)$ and $\check{\Omega}^{0}=\Omega \backslash \bigcup_{j=1}^{m} \Omega_{R_{j} / 32}$. Figure 2 illustrates exemplarily such a partition of the domain. The dashed and the dotted lines indicate (not to scale) the domains $\Omega_{R_{j} / 8}$ and $\Omega_{R_{j}}$, respectively.

Now we will proceed for every corner in the same way. Let the corner $x^{\left(j_{0}\right)}$ be the corner under consideration. We assume for the sake of simplicity but without loss of generality that the corner $x^{\left(j_{0}\right)}$ is located at the origin and $R_{j_{0}}=1$. Furthermore, we suppress the subscript $j_{0}$ in the following, i.e., $\Omega_{R}=\Omega_{R_{j_{0}}}, \Omega_{R / 2}=\Omega_{R_{j_{0}} / 2}$, etc. We divide the domain $\Omega_{R}$ into subsets $\Omega_{J}$,

$$
\Omega_{R}=\bigcup_{J=0}^{I} \Omega_{J}
$$

where $\Omega_{J}=\left\{x: d_{J+1} \leq|x| \leq d_{J}\right\}$ for $J=0, \ldots, I-1$ and $\Omega_{I}=\left\{x:|x| \leq d_{I}\right\}$. The radii $d_{J}$ are set to $2^{-J}$ and the index $I$ is chosen such that

$$
2^{-(I+k+1)} \leq c_{2} h^{1 / \mu} \leq 2^{-(I+k)}
$$

for some fixed $k \in \mathbb{N}_{0}$ and $c_{2}$ from (3.1). Thus, $I \sim \log \frac{1}{h}$ for some $h \leq h_{0}<1$. Obviously, there exists some constant $c_{I} \in \mathbb{R}$ with

$$
c_{2} 2^{k} \leq c_{I} \leq c_{2} 2^{k+1}
$$

such that

$$
d_{I}=2^{-I}=c_{I} h^{1 / \mu} .
$$


For the moment we only assume that the parameter $k$ is chosen such that $c_{I} \geq 1$. It will be exactly specified in the proof of Lemma 3.10 The boundary parts of $\Omega_{J}$ which coincide with the boundary of $\Omega_{R}$ are denoted by $\partial \Omega_{J}^{+}$for $\varphi=\omega$ and by $\partial \Omega_{J}^{-}$for $\varphi=0$. We set $\partial \Omega_{J}^{ \pm}=\partial \Omega_{J}^{+} \cup \partial \Omega_{J}^{-}$. Figure 2 shows such a division. Note that

$$
\Omega_{R / 2}=\bigcup_{J=1}^{I} \Omega_{J}, \Omega_{R / 4}=\bigcup_{J=2}^{I} \Omega_{J}, \Omega_{R / 8}=\bigcup_{J=3}^{I} \Omega_{J}, \text { etc. }
$$

Next we introduce the extended subsets $\Omega_{J}^{\prime}$ for $J \geq 1$ and $\Omega_{J}^{\prime \prime}$ for $J \geq 2$ by

$$
\Omega_{J}^{\prime}=\Omega_{J-1} \cup \Omega_{J} \cup \Omega_{J+1}
$$

and

$$
\Omega_{J}^{\prime \prime}=\Omega_{J-1}^{\prime} \cup \Omega_{J}^{\prime} \cup \Omega_{J+1}^{\prime}
$$

with the obvious modifications for $J=I-1, I$. The boundary parts $\partial \Omega_{J}^{ \pm \prime}$ are analogously defined with respect to $\Omega_{J}^{\prime}$.

Before going into detail let us elucidate the structure of our proof. As we will see on page 51] $L^{2}\left(\Gamma_{R / 16}^{ \pm}\right)$-discretization error estimates are crucial ingredients of the proof of Theorem 3.2 These are established in Lemma 3.12 The proof requires $L^{\infty}\left(\Omega_{J}^{\prime}\right)$-interpolation error estimates (see Lemma 3.7 and Remark 3.8), the weighted finite element error estimate of Lemma 3.10 and some kind of an inverse inequality provided in Lemma 3.11 The proof of Lemma 3.10 relies on a kick back argument, which is established by the special partition of the domain $\Omega_{R}$, the $H^{1}\left(\Omega_{J}\right)$-interpolation error estimates of Lemma [3.7 and local $H^{1}\left(\Omega_{J}\right)$-finite element error estimates provided by Lemma 3.9. Lemma 3.7 and Remark 3.8 are also used in the proof of Lemma 3.9. All these arguments rely on the property that the mesh is quasi-uniform in the strips $\Omega_{J}^{\prime}$ which we are going to prove first.

Lemma 3.6. The element size $h_{T}$ of the elements $T \subset \Omega_{J}^{\prime}$ satisfies

$$
\begin{aligned}
& 2^{-2(1-\mu)} c_{1} h d_{J}^{1-\mu} \leq h_{T} \leq 2^{1-\mu} c_{2} h d_{J}^{1-\mu}, \quad \text { if } 1 \leq J \leq I-2, \\
& c_{1} h^{1 / \mu} \leq h_{T} \leq 2^{2(1-\mu)} c_{2} h d_{I}^{1-\mu}=2^{2(1-\mu)} c_{2} c_{I}^{1-\mu} h^{1 / \mu}, \quad \text { if } J=I, I-1
\end{aligned}
$$

with constants $c_{1}$ and $c_{2}$ from (3.1) and $c_{I}$ from (3.4).

Proof. For any element $T \subset \Omega_{J}^{\prime}$ and $J \leq I-2$ one has $d_{J+2}<r_{T}<d_{J-1}$. Thus, assertion (3.5) follows immediately with $d_{J+2}=2^{-2} d_{J}, d_{J-1}=2 d_{J}$ and the mesh condition (3.1). Assertion (3.6) holds analogously since for any element $T \subset \Omega_{J}$, $J=I, I-1$, one has $0 \leq r_{T} \leq d_{I+2}=2^{2} d_{I}=2^{2} c_{I} h^{1 / \mu}$.

As indicated above we will use a kick back argument in the proof of Lemma 3.10 This depends on the size of the constant $c_{I}$. For that purpose we distinguish between the generic constant $c$ and the constant $c_{I}$ in the following two lemmas.

Lemma 3.7. Let $\mu \in(0,1], v_{1} \in W_{\alpha}^{2, p}\left(\Omega_{J}^{\prime}\right), v_{2} \in W_{\alpha}^{2, \infty}\left(\Omega_{J}^{\prime}\right)$ and $l=0,1$.

(1) For $1 \leq J \leq I-2$ the estimates

$$
\begin{aligned}
\left\|v_{1}-I_{h} v_{1}\right\|_{W^{l, 2}\left(\Omega_{J}\right)} & \leq c h^{2-l} d_{J}^{(2-l)(1-\mu)+1-2 / p-\alpha}\left|v_{1}\right|_{W_{\alpha}^{2, p}\left(\Omega_{J}^{\prime}\right)}, \\
\left\|v_{2}-I_{h} v_{2}\right\|_{L^{\infty}\left(\Omega_{J}\right)} & \leq c h^{2} d_{J}^{2-2 \mu-\alpha}\left|v_{2}\right|_{W_{\alpha}^{2, \infty}\left(\Omega_{J}^{\prime}\right)},
\end{aligned}
$$

are valid for $2 \leq p \leq \infty$ and $\alpha \in \mathbb{R}$. 
(2) Let $\left.1<q \leq \infty, \theta_{l}:=\max \{0,(3-l-2 / p)(1-\mu)-\alpha)\right\}$ and $\theta_{\infty}:=$ $\max \{0,2-2 \mu-\alpha\}$. Then for $J=I, I-1$ the inequalities

$$
\begin{aligned}
\left\|v_{1}-I_{h} v_{1}\right\|_{W^{l, 2}\left(\Omega_{J}\right)} & \leq c c_{I}^{\theta_{l}+1-2 / p} h^{(3-l-\alpha-2 / p) / \mu}\left|v_{1}\right|_{W_{\alpha}^{2, p}\left(\Omega_{J}^{\prime}\right)}, \\
\left\|v_{2}-I_{h} v_{2}\right\|_{L^{\infty}\left(\Omega_{J}\right)} & \leq c c_{I}^{\theta_{\infty}} h^{(2-\alpha) / \mu}\left|v_{2}\right|_{W_{\alpha}^{2, \infty}\left(\Omega_{J}^{\prime}\right)},
\end{aligned}
$$

hold if $\max (2, q) \leq p \leq \infty$ and $-2 / q<\alpha<2-2 / q$.

Proof. We begin with estimating $\left\|v_{1}-I_{h} v_{1}\right\|_{W^{l, 2}\left(\Omega_{J}\right)}, J=0, \ldots, I$, and $l=0,1$. Let $T \in \mathcal{T}_{h}$ be a triangle with $T \cap \Omega_{J} \neq \emptyset$. We first introduce the polynomial $p_{1} \in \mathcal{P}_{1}(T)$ and use the triangle inequality. This yields

$$
\begin{aligned}
\left\|v_{1}-I_{h} v_{1}\right\|_{W^{l, 2}(T)} & =\left\|v_{1}-p_{1}-I_{h}\left(v_{1}-p_{1}\right)\right\|_{W^{l, 2}(T)} \\
& \leq\left\|v_{1}-p_{1}\right\|_{W^{l, 2}(T)}+\left\|I_{h}\left(v_{1}-p_{1}\right)\right\|_{W^{l, 2}(T)} .
\end{aligned}
$$

We can conclude for the first term in (3.11) after the transformation to the reference element $\hat{T}$ and using the embedding $W^{2, q^{\prime}}(\hat{T}) \hookrightarrow W^{l, 2}(\hat{T})$ which holds for $q^{\prime} \geq 1$,

$$
\begin{aligned}
\left\|v_{1}-p_{1}\right\|_{W^{l, 2}(T)} & \leq c|T|^{1 / 2} h_{T}^{-l}\left\|\hat{v}_{1}-\hat{p}_{1}\right\|_{W^{l, 2}(\hat{T})} \\
& \leq c|T|^{1 / 2} h_{T}^{-l}\left\|\hat{v}_{1}-\hat{p}_{1}\right\|_{W^{2, q^{\prime}}(\hat{T})} .
\end{aligned}
$$

To estimate the second term in (3.11) we use an inverse inequality for functions in finite dimensional spaces (only for $l=1$ ) and the embedding $L^{\infty}(\hat{T}) \hookrightarrow L^{2}(\hat{T})$ to get

$$
\begin{aligned}
\left\|I_{h}\left(v_{1}-p_{1}\right)\right\|_{W^{l, 2}(T)} & \leq c|T|^{1 / 2} h_{T}^{-l}\left\|\hat{I}_{h}\left(\hat{v}_{1}-\hat{p}_{1}\right)\right\|_{W^{l, 2}(\hat{T})} \leq c|T|^{1 / 2} h_{T}^{-l}\left\|\hat{I}_{h}\left(\hat{v}_{1}-\hat{p}_{1}\right)\right\|_{L^{2}(\hat{T})} \\
& \leq c|T|^{1 / 2} h_{T}^{-l}\left\|\hat{I}_{h}\left(\hat{v}_{1}-\hat{p}_{1}\right)\right\|_{L^{\infty}(\hat{T})} \leq c|T|^{1 / 2} h_{T}^{-l}\left\|\hat{v}_{1}-\hat{p}_{1}\right\|_{L^{\infty}(\hat{T})} \\
& \leq c|T|^{1 / 2} h_{T}^{-l}\left\|\hat{v}_{1}-\hat{p}_{1}\right\|_{W^{2, q^{\prime}}(\hat{T})} .
\end{aligned}
$$

The last steps hold due to the boundedness of the interpolation operator $I_{h}$ from $L^{\infty}(\hat{T})$ to $L^{\infty}(\hat{T})$ and the embedding $W^{2, q^{\prime}}(\hat{T}) \hookrightarrow L^{\infty}(\hat{T})$, which is valid for $q^{\prime}>1$. The inequalities (3.12) and (3.13) yield together with (3.11) and the Deny-Lions Lemma [10]

$$
\left\|v_{1}-I_{h} v_{1}\right\|_{W^{l, 2}(T)} \leq c|T|^{1 / 2} h_{T}^{-l}\left\|\hat{v}_{1}-\hat{p}_{1}\right\|_{W^{2, q^{\prime}}(\hat{T})} \leq c|T|^{1 / 2} h_{T}^{-l}\left|\hat{v}_{1}\right|_{W^{2, q^{\prime}}(\hat{T})},
$$

which holds for $q^{\prime}>1$. Now we distinguish triangles $T$ with $r_{T}=0$ and $r_{T}>0$. For triangles with $r_{T}=0$ we choose $q^{\prime}=4 q /(2 q+2+\alpha q)>1$ (see Section 9) with some $q>1$ and $-2 / q<\alpha<2-2 / q$. Using the embedding $W_{\alpha}^{2, q}(\hat{T}) \hookrightarrow W^{2, q^{\prime}}(\hat{T})$ according to part 2 of Lemma 2.1 we obtain

$$
\left\|v_{1}-I_{h} v_{1}\right\|_{W^{l, 2}(T)} \leq c|T|^{1 / 2} h_{T}^{-l}\left|\hat{v}_{1}\right|_{W_{\alpha}^{2, q}(\hat{T})} .
$$

The transformation back to the world element yields

$$
\left\|v_{1}-I_{h} v_{1}\right\|_{W^{l, 2}(T)} \leq c h_{T}^{3-l-\alpha-2 / q}\left|v_{1}\right|_{W_{\alpha}^{2, q}(T)} \leq c h^{(3-l-\alpha-2 / q) / \mu}\left|v_{1}\right|_{W_{\alpha}^{2, q}(T)},
$$

since $\hat{r}^{\alpha} \sim h_{T}^{-\alpha} r^{\alpha},|T| \sim h_{T}^{2}$ and $h_{T} \sim h^{1 / \mu}$ (cf. mesh condition (3.1)). In the case that $r_{T}>0$ we easily get from (3.14) for any $q^{\prime}=q>1$ and (3.1)

$$
\begin{aligned}
\left\|v_{1}-I_{h} v_{1}\right\|_{W^{l, 2}(T)} & \leq c h_{T}^{3-l-2 / q}\left|v_{1}\right|_{W^{2, q}(T)} \\
& \leq c h^{3-l-2 / q} r_{T}^{(3-l-2 / q)(1-\mu)-\alpha}\left|v_{1}\right|_{W_{\alpha}^{2, q}(T)} .
\end{aligned}
$$


So far we have derived estimates for $\left\|v_{1}-I_{h} v_{1}\right\|_{W^{l, 2}(T)}$. Next, we consider the domains $\Omega_{J}$. We distinguish between $1 \leq J \leq I-2$ and $J=I-1, I$. In the former case we get from (3.16),

$$
\begin{aligned}
\left\|v_{1}-I_{h} v_{1}\right\|_{W^{l, 2}\left(\Omega_{J}\right)} & \leq\left(\sum_{T \subset \Omega_{J}^{\prime}}\left\|v_{1}-I_{h} v_{1}\right\|_{W^{l, 2}(T)}^{2}\right)^{1 / 2} \\
& \leq c\left(\sum_{T \subset \Omega_{J}^{\prime}}\left(h^{2-l} r_{T}^{(2-l)(1-\mu)-\alpha}\left|v_{1}\right|_{W_{\alpha}^{2,2}(T)}\right)^{2}\right)^{1 / 2} \\
& \leq c h^{2-l} d_{J}^{(2-l)(1-\mu)-\alpha}\left|v_{1}\right|_{W_{\alpha}^{2,2}\left(\Omega_{J}^{\prime}\right)} \\
& \leq c h^{2-l} d_{J}^{(2-l)(1-\mu)+1-2 / p-\alpha}\left|v_{1}\right|_{W_{\alpha}^{2, p}\left(\Omega_{J}^{\prime}\right)},
\end{aligned}
$$

where we used $r_{T} \sim d_{J}$ (cf. Lemma 3.6), the Hölder inequality with some $p \geq 2$ and $\left|\Omega_{J}^{\prime}\right| \sim d_{J}^{2}$ in the last steps. In the case that $J=I-1, I$ we can conclude using (3.15) and (3.16) with some $q>1, p \geq \max (2, q)$ and $-2 / q<\alpha<2-2 / q$,

$$
\begin{aligned}
& \left\|v_{1}-I_{h} v_{1}\right\|_{W^{l, 2}\left(\Omega_{J}\right)} \\
& \leq\left(\sum_{\substack{T \subset \Omega_{J}^{\prime} \\
r_{T}=0}}\left\|v_{1}-I_{h} v_{1}\right\|_{W^{l, 2}(T)}^{2}+\sum_{\substack{T \subset \Omega_{J}^{\prime} \\
r_{T}>0}}\left\|v_{1}-I_{h} v_{1}\right\|_{W^{l, 2}(T)}^{2}\right)^{1 / 2} \\
& \leq c\left(\sum_{\substack{T \subset \Omega_{J}^{\prime} \\
r_{T}=0}}\left(h^{(3-l-\alpha-2 / p) / \mu}\left|v_{1}\right|_{W_{\alpha}^{2, p}(T)}\right)^{2}\right. \\
& \left.+\sum_{\substack{T \subset \Omega_{J}^{\prime} \\
r_{T}>0}}\left(h^{3-l-2 / p} r_{T}^{(3-l-2 / p)(1-\mu)-\alpha}\left|v_{1}\right|_{W_{\alpha}^{2, p}(T)}\right)^{2}\right)^{1 / 2} \\
& \leq c\left(\sum_{\substack{T \subset \Omega_{J}^{\prime} \\
r_{T}=0}}\left(h^{(3-l-\alpha-2 / p) / \mu}\left|v_{1}\right|_{W_{\alpha}^{2, p}(T)}\right)^{2}\right. \\
& \left.+\sum_{\substack{T \subset \Omega_{J}^{\prime} \\
r_{T}>0}}\left(c_{I}^{\theta_{l}} h^{(3-l-\alpha-2 / p) / \mu}\left|v_{1}\right|_{W_{\alpha}^{2, p}(T)}\right)^{2}\right)^{1 / 2} \\
& \leq c c_{I}^{\theta_{l}} h^{(3-l-\alpha-2 / p) / \mu}\left(\sum_{T \subset \Omega_{J}^{\prime}}\left|v_{1}\right|_{W_{\alpha}^{2, p}(T)}^{2}\right)^{1 / 2} \\
& \leq c c_{I}^{\theta_{l}} h^{(3-l-\alpha-2 / p) / \mu}\left|v_{1}\right|_{W_{\alpha}^{2, p}\left(\Omega_{J}^{\prime}\right)}\left(\sum_{T \subset \Omega_{J}^{\prime}} 1\right)^{1 / 2-1 / p},
\end{aligned}
$$


where we used $h^{1 / \mu} \leq r_{T} \leq c d_{I}=c c_{I} h^{1 / \mu}$, if $r_{T}>0$, and the discrete Hölder inequality. Since $\left|\Omega_{J}^{\prime}\right| \sim d_{I}^{2}, d_{I}=c_{I} h^{1 / \mu}$ and $\min _{T \subset \Omega_{J}^{\prime}} h_{T} \sim h^{1 / \mu}$ for $J=I, I-1$ we get that

$$
\left(\sum_{T \subset \Omega_{J}^{\prime}} 1\right)^{1 / 2-1 / p} \leq\left(\frac{\left|\Omega_{J}^{\prime}\right|}{\min _{T \subset \Omega_{J}^{\prime}} h_{T}^{2}}\right)^{1 / 2-1 / p} \leq c\left(c_{I}^{2}\right)^{1 / 2-1 / p}=c c_{I}^{1-2 / p} .
$$

Thus, we obtain for $q>1, p \geq \max (2, q)$ and $-2 / q<\alpha<2-2 / q$,

$$
\left\|v_{1}-I_{h} v_{1}\right\|_{W^{l, 2}\left(\Omega_{J}\right)} \leq c c_{I}^{\theta_{l}+1-2 / p} h^{(3-l-\alpha-2 / p) / \mu}\left|v_{1}\right|_{W_{\alpha}^{2, p}\left(\Omega_{J}^{\prime}\right)} .
$$

It remains to prove the $L^{\infty}$-error estimates. Let $T$ be a triangle with $T \cap \Omega_{J} \neq \emptyset$. As in (3.11) we first insert an arbitrary polynomial $p_{1} \in \mathcal{P}_{1}(T)$. This yields for some $q^{\prime}>1$,

$$
\begin{aligned}
\left\|v_{2}-I_{h} v_{2}\right\|_{L^{\infty}(T)} & \leq\left\|v_{2}-p_{1}\right\|_{L^{\infty}(T)}+\left\|I_{h}\left(v_{2}-p_{1}\right)\right\|_{L^{\infty}(T)} \\
& \leq c\left\|\hat{v}_{2}-\hat{p}_{1}\right\|_{L^{\infty}(\hat{T})} \leq c\left|\hat{v}_{2}\right|_{W_{\alpha}^{2, q^{\prime}}(\hat{T})} ;
\end{aligned}
$$

cf. (3.13) and (3.14). If $r_{T}=0$ we get, as in (3.15), for $q>1$ and $-2 / q<\alpha<2-2 / q$,

$$
\left\|v_{2}-I_{h} v_{2}\right\|_{L^{\infty}(T)} \leq c h_{T}^{2-\alpha-2 / q}\left|v_{2}\right|_{W_{\alpha}^{2, q}(T)} \leq c h^{(2-\alpha) / \mu}\left|v_{2}\right|_{W_{\alpha}^{2, \infty}(T)} .
$$

In the case that $r_{T}>0$ we can conclude, as in (3.16),

$$
\left\|v_{2}-I_{h} v_{2}\right\|_{L^{\infty}(T)} \leq c h_{T}^{2}\left|v_{2}\right|_{W^{2, \infty}(T)} \leq c h^{2} r_{T}^{2-2 \mu-\alpha}\left|v_{2}\right|_{W_{\alpha}^{2, \infty}(T)} .
$$

Now we suppose that $v_{2}-I_{h} v_{2}$ admits its maximum in $\Omega_{J}$ at some point $x_{0} \in \bar{T}_{*} \subset$ $\Omega_{J}^{\prime}$. If $1 \leq J \leq I-2$ we obtain using (3.19) and $r_{T} \sim d_{J}$,

$$
\begin{aligned}
\left\|v_{2}-I_{h} v_{2}\right\|_{L^{\infty}\left(\Omega_{J}\right)} & =\left\|v_{2}-I_{h} v_{2}\right\|_{L^{\infty}\left(T_{*}\right)} \leq c h^{2} d_{J}^{2-2 \mu-\alpha}\left|v_{2}\right|_{W_{\alpha}^{2, \infty}\left(T_{*}\right)} \\
& \leq \operatorname{ch}^{2} d_{J}^{2-2 \mu-\alpha}\left|v_{2}\right|_{W_{\alpha}^{2, \infty}\left(\Omega_{J}^{\prime}\right)} .
\end{aligned}
$$

In the case that $J=I-1, I$ we get for $r_{T_{*}}=0$, according to (3.18),

$$
\left\|v_{2}-I_{h} v_{2}\right\|_{L^{\infty}\left(\Omega_{J}\right)} \leq c h_{T_{*}}^{2-\alpha-2 / q}\left|v_{2}\right|_{W_{\alpha}^{2, q}\left(T_{*}\right)} \leq c h_{T_{*}}^{2-\alpha}\left|v_{2}\right|_{W_{\alpha}^{2, \infty}\left(T_{*}\right)} \leq c h_{T_{*}}^{2-\alpha}\left|v_{2}\right|_{W_{\alpha}^{2, \infty}\left(\Omega_{J}^{\prime}\right)},
$$

which holds for some $q>1$ and $-2 / q<\alpha<2-2 / q$. Since $h_{T_{*}} \sim h^{1 / \mu}$ we can continue with

$$
\left\|v_{2}-I_{h} v_{2}\right\|_{L^{\infty}\left(\Omega_{J}\right)} \leq \mathrm{ch}^{(2-\alpha) / \mu}\left|v_{2}\right|_{W_{\alpha}^{2, \infty}\left(\Omega_{J}^{\prime}\right)} .
$$

In the case that $r_{T_{*}}>0$ we can conclude analogously to (3.17) using (3.19),

$$
\begin{aligned}
\left\|v_{2}-I_{h} v_{2}\right\|_{L^{\infty}\left(\Omega_{J}\right)} & =\left\|v_{2}-I_{h} v_{2}\right\|_{L^{\infty}\left(T_{*}\right)} \leq c h_{T_{*}}^{2}\left|v_{2}\right|_{W^{2, \infty}\left(T_{*}\right)} \\
& \leq c h^{2} r_{T_{*}}^{2-2 \mu-\alpha}\left|v_{2}\right|_{W_{\alpha}^{2, \infty}\left(T_{*}\right)} \leq c c_{I}^{\theta_{\infty}} h^{2} h^{(2-2 \mu-\alpha) / \mu}\left|v_{2}\right|_{W_{\alpha}^{2, \infty}\left(\Omega_{J}^{\prime}\right)} \\
& =\left.c c_{I}^{\theta \infty} h^{(2-\alpha) / \mu}\right|_{\left.V_{2}\right|_{W_{\alpha}^{2, \infty}\left(\Omega_{J}^{\prime}\right)} .}
\end{aligned}
$$

Remark 3.8. The inequalities (3.7) - 3.10) hold as well if we replace $\Omega_{J}$ with $\Omega_{J}^{\prime}$ and $\Omega_{J}^{\prime}$ with $\Omega_{J}^{\prime \prime}$, respectively. In that case we have to distinguish $2 \leq J \leq I-3$ and $J=I-2, I-1, I$.

Lemma 3.9. Let $\mu \in(0,1]$ and $y \in W_{\alpha}^{2, \infty}\left(\Omega_{R}\right)$. 
(1) For $2 \leq J \leq I-3$ the estimate

$$
\left\|y-y_{h}\right\|_{H^{1}\left(\Omega_{J}\right)} \leq c\left(h d_{J}^{2-\mu-\alpha}|y|_{W_{\alpha}^{2, \infty}\left(\Omega_{J}^{\prime \prime}\right)}+d_{J}^{-1}\left\|y-y_{h}\right\|_{L^{2}\left(\Omega_{J}^{\prime}\right)}\right)
$$

is valid for $\alpha \in \mathbb{R}$.

(2) Let $1<q \leq \infty$. Then for $J \geq I-2$ the inequality

$$
\left\|y-y_{h}\right\|_{H^{1}\left(\Omega_{J}\right)} \leq c\left(c_{I}^{5} h^{(2-\alpha) / \mu}|y|_{W_{\alpha}^{2, \infty}\left(\Omega_{J}^{\prime \prime}\right)}+d_{J}^{-1}\left\|y-y_{h}\right\|_{L^{2}\left(\Omega_{J}^{\prime}\right)}\right)
$$

holds true for $-2 / q<\alpha<2-2 / q$.

Proof. The proof relies on local finite element error estimates stated in [9] and on the interpolation error estimates given in Lemma 3.7 For $J=0, \ldots, I$ we get from Theorem 3.4 of [9],

$$
\left\|y-y_{h}\right\|_{H^{1}\left(\Omega_{J}\right)} \leq c\left(\left\|y-I_{h} y\right\|_{H^{1}\left(\Omega_{J}^{\prime}\right)}+d_{J}^{-1}\left\|y-I_{h} y\right\|_{L^{2}\left(\Omega_{J}^{\prime}\right)}+d_{J}^{-1}\left\|y-y_{h}\right\|_{L^{2}\left(\Omega_{J}^{\prime}\right)}\right),
$$

where the constant $c$ does not depend on $c_{I}$. In the case that $2 \leq J \leq I-3$ one gets with Lemma 3.7 and Remark 3.8

$$
\begin{gathered}
\left\|y-y_{h}\right\|_{H^{1}\left(\Omega_{J}\right)} \leq c( \\
h d_{J}^{2-\mu-\alpha}|y|_{W_{\alpha}^{2, \infty}\left(\Omega_{J}^{\prime \prime}\right)}+h^{2} d_{J}^{2-2 \mu-\alpha}|y|_{W_{\alpha}^{2, \infty}\left(\Omega_{J}^{\prime \prime}\right)} \\
\left.+d_{J}^{-1}\left\|y-y_{h}\right\|_{L^{2}\left(\Omega_{J}^{\prime}\right)}\right) .
\end{gathered}
$$

Since $h d_{J}^{-\mu} \leq h d_{I}^{-\mu}=c_{I}^{-\mu} \leq 1$ we arrive at

$$
\left\|y-y_{h}\right\|_{H^{1}\left(\Omega_{J}\right)} \leq c\left(h d_{J}^{2-\mu-\alpha}|y|_{W_{\alpha}^{2, \infty}\left(\Omega_{J}^{\prime \prime}\right)}+d_{J}^{-1}\left\|y-y_{h}\right\|_{L^{2}\left(\Omega_{J}^{\prime}\right)}\right) .
$$

This is the first inequality of the assertion. For $J \geq I-2$ we proceed in an analogous way. But now we use the interpolation error estimates from Lemma 3.7, with regard to Remark 3.8 which are stated there for domains close to or at the corner. Let $\theta_{l}:=\max \{0,(3-l)(1-\mu)-\alpha\}$ for $l=0,1$. By this we obtain

$$
\begin{aligned}
\left\|y-y_{h}\right\|_{H^{1}\left(\Omega_{J}\right)} \leq & c\left(c_{I}^{\theta_{1}+1} h^{(2-\alpha) / \mu}|y|_{W_{\alpha}^{2, \infty}\left(\Omega_{J}^{\prime \prime}\right)}+c_{I}^{\theta_{0}+1} d_{J}^{-1} h^{(3-\alpha) / \mu}|y|_{W_{\alpha}^{2, \infty}\left(\Omega_{J}^{\prime \prime}\right)}\right. \\
& \left.\quad+d_{J}^{-1}\left\|y-y_{h}\right\|_{L^{2}\left(\Omega_{J}^{\prime}\right)}\right) \\
\leq & c\left(\left(c_{I}^{\theta_{1}+1}+c_{I}^{\theta_{0}}\right) h^{(2-\alpha) / \mu}|y|_{W_{\alpha}^{2, \infty}\left(\Omega_{J}^{\prime \prime}\right)}+d_{J}^{-1}\left\|y-y_{h}\right\|_{L^{2}\left(\Omega_{J}^{\prime}\right)}\right) \\
\leq & c\left(c_{I}^{5} h^{(2-\alpha) / \mu}|y|_{W_{\alpha}^{2, \infty}\left(\Omega_{J}^{\prime \prime}\right)}+d_{J}^{-1}\left\|y-y_{h}\right\|_{L^{2}\left(\Omega_{J}^{\prime}\right)}\right)
\end{aligned}
$$

where we used $d_{J}^{-1} h^{1 / \mu} \leq d_{I}^{-1} h^{1 / \mu}=c_{I}^{-1}, \theta_{1} \leq 4$ and $\theta_{0} \leq 5$ in the last steps.

Lemma 3.10. Let $1<q \leq \infty$. Then for $y \in W_{\gamma}^{2, \infty}\left(\Omega_{R}\right), \gamma \leq 5 / 2-2 \mu$ and $-2 / q<\gamma<2-2 / q$ the inequality

$$
\left\|\left(r+d_{I}\right)^{-1 / 2}\left(y-y_{h}\right)\right\|_{L^{2}\left(\Omega_{R / 8}\right)} \leq c\left(h^{2}|\ln h|^{1 / 2}|y|_{W_{\gamma}^{2, \infty}\left(\Omega_{R}\right)}+\left\|y-y_{h}\right\|_{L^{2}\left(\Omega_{R}\right)}\right)
$$

holds.

Proof. We define the weight function $\sigma=r+d_{I}$. Furthermore, let $\chi$ be the characteristic function, which is equal to one in $\Omega_{R / 8}$ and equal to zero in $\Omega_{R} \backslash \operatorname{cl}\left(\Omega_{R / 8}\right)$. Next, we introduce the boundary value problem

$$
\begin{aligned}
-\Delta w+w & =\sigma^{-1}\left(y-y_{h}\right) \chi & & \text { in } \Omega_{R}, \\
\partial_{n} w & =0 & & \text { on } \partial \Omega_{R}
\end{aligned}
$$


with its weak formulation

$$
a_{\Omega_{R}}(\varphi, w)=\left(\sigma^{-1}\left(y-y_{h}\right) \chi, \varphi\right)_{L^{2}\left(\Omega_{R}\right)} \quad \forall \varphi \in H^{1}\left(\Omega_{R}\right),
$$

where the bilinear form $a_{\Omega_{R}}: H^{1}\left(\Omega_{R}\right) \times H^{1}\left(\Omega_{R}\right) \rightarrow \mathbb{R}$ is defined by

$$
a_{\Omega_{R}}(\varphi, w)=\int_{\Omega_{R}}(\nabla \varphi \cdot \nabla w+\varphi w) \mathrm{d} x .
$$

Since $r\left(r+d_{I}\right)^{-2} \leq r^{-1}$ and $y-y_{h} \in H^{1}(\Omega)$ we can conclude, using part 1 of Lemma 2.1, that

$$
\begin{aligned}
\left\|\sigma^{-1}\left(y-y_{h}\right) \chi\right\|_{W_{1 / 2}^{0,2}\left(\Omega_{R}\right)} & \leq\left\|\sigma^{-1}\left(y-y_{h}\right)\right\|_{W_{1 / 2}^{0,2}\left(\Omega_{R}\right)} \leq\left\|y-y_{h}\right\|_{W_{-1 / 2}^{0,2}\left(\Omega_{R}\right)} \\
& \leq\left\|y-y_{h}\right\|_{H^{1}\left(\Omega_{R}\right)}
\end{aligned}
$$

this means for the right-hand side of (3.20) that $\sigma^{-1}\left(y-y_{h}\right) \chi \in W_{1 / 2}^{0,2}\left(\Omega_{R}\right)$. Thus, we get according to Lemma 2.3 that the solution $w$ belongs to $W_{1 / 2}^{2,2}\left(\Omega_{R}\right)$, since $1>1 / 2>1-\lambda=1-\pi / \omega$ for every angle $\omega$ in $(0,2 \pi)$. Moreover, if we use the inequality $r<r+d_{I}$ we obtain the validity of the a priori estimate

$$
\|w\|_{W_{1 / 2}^{2,2}\left(\Omega_{R}\right)} \leq c\left\|\sigma^{-1}\left(y-y_{h}\right)\right\|_{W_{1 / 2}^{0,2}\left(\Omega_{R / 8}\right)} \leq c\left\|\sigma^{-1 / 2}\left(y-y_{h}\right)\right\|_{L^{2}\left(\Omega_{R / 8}\right)} .
$$

Using part 1 of Lemma 2.1 we can also show that

$$
\|w\|_{H^{1}\left(\Omega_{R}\right)}=\|w\|_{W_{0}^{1,2}\left(\Omega_{R}\right)} \leq c\|w\|_{W_{1 / 2}^{2,2}\left(\Omega_{R}\right)} \leq c\left\|\sigma^{-1 / 2}\left(y-y_{h}\right)\right\|_{L^{2}\left(\Omega_{R / 8}\right)} .
$$

Now, let $\eta$ be an infinitely differentiable function in $\operatorname{cl}(\Omega)$, which is equal to one in $\Omega_{R / 8}$, supp $\eta \subset \Omega_{R / 4}$ and $\partial_{n} \eta=0$ on $\partial \Omega_{R}$. By setting $\varphi=\eta v$ in (3.21) with some $v \in H^{1}\left(\Omega_{R}\right)$ one can show that $\tilde{w}=\eta w$ fulfills the equation

$$
a_{\Omega_{R}}(v, \tilde{w})=\left(\eta \sigma^{-1}\left(y-y_{h}\right) \chi-\Delta \eta w-2 \nabla \eta \cdot \nabla w, v\right)_{L^{2}\left(\Omega_{R}\right)} \quad \forall v \in H^{1}\left(\Omega_{R}\right) .
$$

By this we get

$$
\begin{aligned}
\| \sigma^{-1 / 2} & \left(y-y_{h}\right) \|_{L^{2}\left(\Omega_{R / 8}\right)}^{2}=\left(\eta \sigma^{-1}\left(y-y_{h}\right) \chi, y-y_{h}\right)_{L^{2}\left(\Omega_{R}\right)} \\
& =a_{\Omega_{R}}\left(y-y_{h}, \tilde{w}\right)+\left(\Delta \eta w, y-y_{h}\right)_{L^{2}\left(\Omega_{R}\right)}+2\left(\nabla \eta \cdot \nabla w, y-y_{h}\right)_{L^{2}\left(\Omega_{R}\right)} \\
& \leq a_{\Omega_{R}}\left(y-y_{h}, \tilde{w}\right)+\left(\|\Delta \eta w\|_{L^{2}\left(\Omega_{R}\right)}+2\|\nabla \eta \cdot \nabla w\|_{L^{2}\left(\Omega_{R}\right)}\right)\left\|y-y_{h}\right\|_{L^{2}\left(\Omega_{R}\right)} \\
& \leq a_{\Omega_{R}}\left(y-y_{h}, \tilde{w}\right)+c\|w\|_{H^{1}\left(\Omega_{R}\right)}\left\|y-y_{h}\right\|_{L^{2}\left(\Omega_{R}\right)} \\
& \leq a_{\Omega_{R}}\left(y-y_{h}, \tilde{w}\right)+c\left\|\sigma^{-1 / 2}\left(y-y_{h}\right)\right\|_{L^{2}\left(\Omega_{R / 8}\right)}\left\|y-y_{h}\right\|_{L^{2}\left(\Omega_{R}\right)},
\end{aligned}
$$

where we used the Cauchy-Schwarz inequality and (3.23) in the last steps. It remains to estimate the first term in (3.24). Since $\tilde{w}$ is equal to zero in $\Omega_{R} \backslash \operatorname{cl}\left(\Omega_{R / 4}\right)$ we can use the Galerkin orthogonality of $y-y_{h}$, i.e., $a_{\Omega_{R}}\left(y-y_{h}, I_{h} \tilde{w}\right)=a(y-$ $\left.y_{h}, I_{h} \tilde{w}\right)=0$. This yields together with an application of the Cauchy-Schwarz inequality

$$
\begin{aligned}
a_{\Omega_{R}}\left(y-y_{h}, \tilde{w}\right) & =a_{\Omega_{R}}\left(y-y_{h}, \tilde{w}-I_{h} \tilde{w}\right) \\
& \leq c \sum_{J=2}^{I}\left\|y-y_{h}\right\|_{H^{1}\left(\Omega_{J}\right)}\left\|\tilde{w}-I_{h} \tilde{w}\right\|_{H^{1}\left(\Omega_{J}\right)} .
\end{aligned}
$$

Remember that $\tilde{w}-I_{h} \tilde{w} \equiv 0$ in $\Omega_{J}$ for $J=0,1$. Now each term on the right-hand side of (3.25) is estimated separately. We distinguish between $2 \leq J \leq I-3$ and 
$J=I, I-1, I-2$ as it has already been done in the previous lemmas. We get for $2 \leq J \leq I-3$ with Lemma 3.9

$$
\left\|y-y_{h}\right\|_{H^{1}\left(\Omega_{J}\right)} \leq c\left(h d_{J}^{2-\mu-\gamma}|y|_{W_{\gamma}^{2, \infty}\left(\Omega_{J}^{\prime \prime}\right)}+d_{J}^{-1}\left\|y-y_{h}\right\|_{L^{2}\left(\Omega_{J}^{\prime}\right)}\right)
$$

and with Lemma 3.7 with $\alpha=1 / 2$,

$$
\left\|\tilde{w}-I_{h} \tilde{w}\right\|_{H^{1}\left(\Omega_{J}\right)} \leq \operatorname{chd} d_{J}^{1 / 2-\mu}|\tilde{w}|_{W_{1 / 2}^{2,2}\left(\Omega_{J}^{\prime}\right)},
$$

By means of these two estimates one can conclude for $2 \leq J \leq I-3$,

$$
\begin{aligned}
& \left\|y-y_{h}\right\|_{H^{1}\left(\Omega_{J}\right)}\left\|\tilde{w}-I_{h} \tilde{w}\right\|_{H^{1}\left(\Omega_{J}\right)} \\
& \quad \leq c\left(h^{2} d_{J}^{5 / 2-2 \mu-\gamma}|y|_{W_{\gamma}^{2, \infty}\left(\Omega_{J}^{\prime \prime}\right)}+h d_{J}^{-\mu}\left\|d_{J}^{-1 / 2}\left(y-y_{h}\right)\right\|_{L^{2}\left(\Omega_{J}^{\prime}\right)}\right)|\tilde{w}|_{W_{1 / 2}^{2,2}\left(\Omega_{J}^{\prime}\right)} \\
& \quad \leq c\left(h^{2} d_{J}^{5 / 2-2 \mu-\gamma}|y|_{W_{\gamma}^{2, \infty}\left(\Omega_{J}^{\prime \prime}\right)}+c_{I}^{-\mu}\left\|d_{J}^{-1 / 2}\left(y-y_{h}\right)\right\|_{L^{2}\left(\Omega_{J}^{\prime}\right)}\right)|\tilde{w}|_{W_{1 / 2}^{2,2}\left(\Omega_{J}^{\prime}\right)},
\end{aligned}
$$

where we used $h d_{J}^{-\mu} \leq h d_{I}^{-\mu}=c_{I}^{-\mu}$. For $J=I, I-1, I-2$ we get from Lemma 3.9 for $1<q \leq \infty$ and $-2 / q<\gamma<2-2 / q$,

$$
\left\|y-y_{h}\right\|_{H^{1}\left(\Omega_{J}\right)} \leq c\left(c_{I}^{5} h^{(2-\gamma) / \mu}|y|_{W_{\gamma}^{2, \infty}\left(\Omega_{J}^{\prime \prime}\right)}+d_{J}^{-1}\left\|y-y_{h}\right\|_{L^{2}\left(\Omega_{J}^{\prime}\right)}\right)
$$

and from Lemma 3.7 with $\alpha=1 / 2$

$$
\left\|\tilde{w}-I_{h} \tilde{w}\right\|_{H^{1}\left(\Omega_{J}\right)} \leq c c_{I}^{\max \{0,1 / 2-\mu\}} h^{1 / 2 \mu}|\tilde{w}|_{W_{1 / 2}^{2,2}\left(\Omega_{J}^{\prime}\right)} .
$$

We can combine the last two estimates to arrive at

$$
\begin{aligned}
\| y & -y_{h}\left\|_{H^{1}\left(\Omega_{J}\right)}\right\| \tilde{w}-I_{h} \tilde{w} \|_{H^{1}\left(\Omega_{J}\right)} \leq c\left(c_{I}^{11 / 2} h^{(5 / 2-\gamma) / \mu}|y|_{W_{\gamma}^{2, \infty}\left(\Omega_{J}^{\prime \prime}\right)}\right. \\
& \left.+c_{I}^{\max \{0,1 / 2-\mu\}}\left(h^{1 / \mu} d_{J}^{-1}\right)^{1 / 2}\left\|d_{J}^{-1 / 2}\left(y-y_{h}\right)\right\|_{L^{2}\left(\Omega_{J}^{\prime}\right)}\right)|\tilde{w}|_{W_{1 / 2}^{2,2}\left(\Omega_{J}^{\prime}\right)} \\
& \leq c\left(c_{I}^{11 / 2} h^{(5 / 2-\gamma) / \mu}|y|_{W_{\gamma}^{2, \infty}\left(\Omega_{J}^{\prime \prime}\right)}\right. \\
& \left.+c_{I}^{\max \{-1 / 2,-\mu\}}\left\|d_{J}^{-1 / 2}\left(y-y_{h}\right)\right\|_{L^{2}\left(\Omega_{J}^{\prime}\right)}\right)|\tilde{w}|_{W_{1 / 2}^{2,2}\left(\Omega_{J}^{\prime}\right)},
\end{aligned}
$$

where we used $\max \{0,1 / 2-\mu\}<1 / 2$ and $h^{1 / \mu} d_{J}^{-1} \leq h^{1 / \mu} d_{I}^{-1}=c_{I}^{-1}$. Let $\theta:=$ $\max \{-1 / 2,-\mu\}$. Inserting the inequalities (3.26) and (3.27) into (3.25) yields

$$
\begin{aligned}
& a_{\Omega_{R}}\left(y-y_{h}, \tilde{w}-I_{h} \tilde{w}\right) \\
& \quad \leq c \sum_{J=2}^{I-3}\left(h^{2} d_{J}^{5 / 2-2 \mu-\gamma}|y|_{W_{\gamma}^{2, \infty}\left(\Omega_{J}^{\prime \prime}\right)}+c_{I}^{-\mu}\left\|d_{J}^{-1 / 2}\left(y-y_{h}\right)\right\|_{L^{2}\left(\Omega_{J}^{\prime}\right)}\right)|\tilde{w}|_{W_{1 / 2}^{2,2}\left(\Omega_{J}^{\prime}\right)} \\
& \quad+c \sum_{J=I-2}^{I}\left(c_{I}^{11 / 2} h^{(5 / 2-\gamma) / \mu}|y|_{W_{\gamma}^{2, \infty}\left(\Omega_{J}^{\prime \prime}\right)}+c_{I}^{\theta}\left\|d_{J}^{-1 / 2}\left(y-y_{h}\right)\right\|_{L^{2}\left(\Omega_{J}^{\prime}\right)}\right)|\tilde{w}|_{W_{1 / 2}^{2,2}\left(\Omega_{J}^{\prime}\right)} .
\end{aligned}
$$

If we additionally set $\gamma \leq 5 / 2-2 \mu$, using $c_{I}^{-\mu}<c_{I}^{\theta}$ and $d_{J}^{-1} \leq c \sigma^{-1}$, we can conclude

$$
\begin{aligned}
& a_{\Omega_{R}}\left(y-y_{h}, \tilde{w}-I_{h} \tilde{w}\right) \\
& \quad \leq c \sum_{J=2}^{I}\left(c_{I}^{11 / 2} h^{2}|y|_{W_{\gamma}^{2, \infty}\left(\Omega_{J}^{\prime \prime}\right)}+c_{I}^{\theta}\left\|\sigma^{-1 / 2}\left(y-y_{h}\right)\right\|_{L^{2}\left(\Omega_{J}^{\prime}\right)}\right)|\tilde{w}|_{W_{1 / 2}^{2,2}\left(\Omega_{J}^{\prime}\right)} .
\end{aligned}
$$


Now we get with $\sum_{J=2}^{I} 1 \sim|\ln h|$ and the discrete Cauchy-Schwarz inequality

$$
\begin{aligned}
& a_{\Omega_{R}}\left(y-y_{h}, \tilde{w}-I_{h} \tilde{w}\right) \\
& \quad \leq c\left(c_{I}^{11 / 2} h^{2}|\ln h|^{1 / 2}|y|_{W_{\gamma}^{2, \infty}\left(\Omega_{R}\right)}+c_{I}^{\theta}\left\|\sigma^{-1 / 2}\left(y-y_{h}\right)\right\|_{L^{2}\left(\Omega_{R}\right)}\right)|\tilde{w}|_{W_{1 / 2}^{2,2}\left(\Omega_{R}\right)} .
\end{aligned}
$$

Since $|\tilde{w}|_{W_{1 / 2}^{2,2}\left(\Omega_{R}\right)} \leq c\|w\|_{W_{1 / 2}^{2,2}\left(\Omega_{R}\right)}$ we can apply the a priori estimate (3.22), which yields

$$
\begin{aligned}
& a_{\Omega_{R}}\left(y-y_{h}, \tilde{w}-I_{h} \tilde{w}\right) \leq c\left(c_{I}^{11 / 2} h^{2}|\ln h|^{1 / 2}|y|_{W_{\gamma}^{2, \infty}\left(\Omega_{R}\right)}\right. \\
& \left.\quad+c_{I}^{\theta}\left\|\sigma^{-1 / 2}\left(y-y_{h}\right)\right\|_{L^{2}\left(\Omega_{R}\right)}\right)\left\|\sigma^{-1 / 2}\left(y-y_{h}\right)\right\|_{L^{2}\left(\Omega_{R / 8}\right)} .
\end{aligned}
$$

By inserting (3.28) into (3.24) and dividing by $\left\|\sigma^{-1 / 2}\left(y-y_{h}\right)\right\|_{L^{2}\left(\Omega_{R / 8}\right)}$ we obtain

$$
\begin{aligned}
& \left\|\sigma^{-1 / 2}\left(y-y_{h}\right)\right\|_{L^{2}\left(\Omega_{R / 8}\right)} \\
& \quad \leq c\left(c_{I}^{11 / 2} h^{2}|\ln h|^{1 / 2}|y|_{W_{\gamma}^{2, \infty}\left(\Omega_{R}\right)}+c_{I}^{\theta}\left\|\sigma^{-1 / 2}\left(y-y_{h}\right)\right\|_{L^{2}\left(\Omega_{R}\right)}+\left\|y-y_{h}\right\|_{L^{2}\left(\Omega_{R}\right)}\right) \\
& \quad \leq c\left(c_{I}^{11 / 2} h^{2}|\ln h|^{1 / 2}|y|_{W_{\gamma}^{2, \infty}\left(\Omega_{R}\right)}+c_{I}^{\theta}\left\|\sigma^{-1 / 2}\left(y-y_{h}\right)\right\|_{L^{2}\left(\Omega_{R / 8}\right)}+c_{I}^{\theta}\left\|y-y_{h}\right\|_{L^{2}\left(\Omega_{R}\right)}\right),
\end{aligned}
$$

where we used $\sigma^{-1 / 2}=\left(r+d_{I}\right)^{-1 / 2} \leq r^{-1 / 2} \leq(R / 8)^{-1 / 2} \leq c$ if $r \geq R / 8$. Finally, we get

$$
\begin{aligned}
& \left(1-c c_{I}^{\theta}\right)\left\|\sigma^{-1 / 2}\left(y-y_{h}\right)\right\|_{L^{2}\left(\Omega_{R / 8}\right)} \\
& \quad \leq c\left(c_{I}^{11 / 2} h^{2}|\ln h|^{1 / 2}|y|_{W_{\gamma}^{2, \infty}\left(\Omega_{R}\right)}+c_{I}^{\theta}\left\|y-y_{h}\right\|_{L^{2}\left(\Omega_{R}\right)}\right) .
\end{aligned}
$$

If one has chosen the parameter $k$ in (3.3) large enough such that

$$
c c_{I}^{\theta}=c c_{I}^{\max \{-1 / 2,-\mu\}} \leq c\left(c_{2} 2^{k}\right)^{\max \{-1 / 2,-\mu\}}<1,
$$

then the desired result follows.

In the remainder of this section the constant $c_{I}$ is hidden in the generic constant $c$.

Lemma 3.11. For $v_{h} \in V_{h}$ and $1 \leq p \leq \infty$ there exists a constant $c>0$ such that

$$
\begin{array}{ll}
\left\|v_{h}\right\|_{L^{p}\left(\partial \Omega_{J}^{ \pm}\right)} \leq c h^{-1 / p} d_{J}^{-(1-\mu) / p}\left\|v_{h}\right\|_{L^{p}\left(\Omega_{J}^{\prime}\right)} & \text { for } 1 \leq J \leq I-2, \\
\left\|v_{h}\right\|_{L^{p}\left(\partial \Omega_{J}^{ \pm}\right)} \leq c h^{-1 /(p \mu)}\left\|v_{h}\right\|_{L^{p}\left(\Omega_{J}^{\prime}\right)} & \text { for } J=I-1, I .
\end{array}
$$

Proof. Let $E \subset \partial \Omega_{J}^{ \pm \prime}$, and let $T \subset \Omega_{J}^{\prime}$ be the corresponding triangle. By an affine change of variables to the reference edge $\hat{E}$ and reference triangle $\hat{T}$, respectively, using the continuity of $\hat{v}_{h}$ on $\operatorname{cl}(\hat{T})$ and the norm equivalence in finite dimensional spaces we obtain

$$
\begin{aligned}
\left\|v_{h}\right\|_{L^{p}(E)} & \leq c h_{T}^{1 / p}\left\|\hat{v}_{h}\right\|_{L^{p}(\hat{E})} \leq c h_{T}^{1 / p}\left\|\hat{v}_{h}\right\|_{L^{\infty}(\hat{E})} \leq c h_{T}^{1 / p}\left\|\hat{v}_{h}\right\|_{L^{\infty}(\hat{T})} \\
& \leq c h_{T}^{1 / p}\left\|\hat{v}_{h}\right\|_{L^{p}(\hat{T})} \leq c h_{T}^{-1 / p}\left\|v_{h}\right\|_{L^{p}(T)} .
\end{aligned}
$$

Now we can sum up to get

$$
\begin{aligned}
\left\|v_{h}\right\|_{L^{p}\left(\partial \Omega_{J}^{ \pm}\right)}^{p} & \leq \sum_{E \subset \partial \Omega_{J}^{ \pm}}\left\|v_{h}\right\|_{L^{p}(E)}^{p} \leq c \sum_{T \subset \Omega_{J}^{\prime}}\left(h_{T}^{-1}\left\|v_{h}\right\|_{L^{p}(T)}^{p}\right) \\
& \leq c \min _{T \subset \Omega_{J}^{\prime}} h_{T}^{-1} \sum_{T \subset \Omega_{J}^{\prime}}\left\|v_{h}\right\|_{L^{p}(T)}^{p} .
\end{aligned}
$$

One can conclude the desired result with Lemma 3.6. 
Lemma 3.12. Let $0 \leq \delta \leq 1 / 2$ and $1<q \leq \infty$. Then for $y \in W_{\gamma}^{2, \infty}\left(\Omega_{R}\right)$, $\gamma \leq 2+\delta-2 \mu$ and $-2 / q<\gamma<2-2 / q$ the estimate

$$
\left\|y-y_{h}\right\|_{L^{2}\left(\Gamma_{R / 16}^{ \pm}\right)} \leq c\left(h^{2}|\ln h|^{1+\delta}|y|_{W_{\gamma}^{2, \infty}\left(\Omega_{R}\right)}+\left\|y-y_{h}\right\|_{L^{2}\left(\Omega_{R}\right)}\right)
$$

is valid.

Proof. Note that $\Gamma_{R / 16}^{ \pm}=\bigcup_{J=4}^{I} \partial \Omega_{J}^{ \pm}$. It holds for $J=I-1, I$ that

$$
\begin{aligned}
\left\|y-y_{h}\right\|_{L^{2}\left(\partial \Omega_{J}^{ \pm}\right)} & \leq\left\|y-I_{h} y\right\|_{L^{2}\left(\partial \Omega_{J}^{ \pm}\right)}+\left\|I_{h} y-y_{h}\right\|_{L^{2}\left(\partial \Omega_{J}^{ \pm}\right)} \\
& \leq c d_{J}^{1 / 2}\left\|y-I_{h} y\right\|_{L^{\infty}\left(\partial \Omega_{J}^{ \pm}\right)}+\left\|I_{h} y-y_{h}\right\|_{L^{2}\left(\partial \Omega_{J}^{ \pm}\right)},
\end{aligned}
$$

where we have used $\left|\partial \Omega_{J}^{ \pm}\right| \sim d_{J}$. The continuity of $y-I_{h} y$ on $\operatorname{cl}\left(\Omega_{J}\right)$ and Lemma 3.11 with $p=2$ yields

$$
\left\|y-y_{h}\right\|_{L^{2}\left(\partial \Omega_{J}^{ \pm}\right)} \leq c d_{J}^{1 / 2}\left\|y-I_{h} y\right\|_{L^{\infty}\left(\Omega_{J}\right)}+c h^{-1 /(2 \mu)}\left\|I_{h} y-y_{h}\right\|_{L^{2}\left(\Omega_{J}^{\prime}\right)} .
$$

Since $d_{J} \sim h^{1 / \mu}$ for $J=I-1, I$ and $\left|\Omega_{J}^{\prime}\right| \sim d_{J}^{2}$ we can proceed with

$$
\begin{aligned}
& \left\|y-y_{h}\right\|_{L^{2}\left(\partial \Omega_{J}^{ \pm}\right)} \\
& \quad \leq c d_{J}^{1 / 2}\left\|y-I_{h} y\right\|_{L^{\infty}\left(\Omega_{J}\right)}+c d_{J}^{-1 / 2}\left\|y-I_{h} y\right\|_{L^{2}\left(\Omega_{J}^{\prime}\right)}+c d_{J}^{-1 / 2}\left\|y-y_{h}\right\|_{L^{2}\left(\Omega_{J}^{\prime}\right)} \\
& \quad \leq c d_{J}^{1 / 2}\left\|y-I_{h} y\right\|_{L^{\infty}\left(\Omega_{J}^{\prime}\right)}+c d_{J}^{-1 / 2}\left\|y-y_{h}\right\|_{L^{2}\left(\Omega_{J}^{\prime}\right)} .
\end{aligned}
$$

Next we consider the case $4 \leq J \leq I-2$. Again we use $\left|\partial \Omega_{J}^{ \pm}\right| \sim d_{J}$ and the continuity of $y-I_{h} y$ on $\operatorname{cl}\left(\Omega_{J}\right)$. Thus we can write

$$
\left\|y-y_{h}\right\|_{L^{2}\left(\partial \Omega_{J}^{ \pm}\right)} \leq c d_{J}^{1 / 2}\left\|y-y_{h}\right\|_{L^{\infty}\left(\partial \Omega_{J}^{ \pm}\right)} \leq c d_{J}^{1 / 2}\left\|y-y_{h}\right\|_{L^{\infty}\left(\Omega_{J}\right)} .
$$

Since each subdomain $\Omega_{J}^{\prime}$ has a positive distance to the corner for $4 \leq J \leq I-2$, we can use Theorem 10.1 in 32 with $s=0$ to get

$$
\left\|y-y_{h}\right\|_{L^{2}\left(\partial \Omega_{J}^{ \pm}\right)} \leq c d_{J}^{1 / 2}|\ln h|\left\|y-I_{h} y\right\|_{L^{\infty}\left(\Omega_{J}^{\prime}\right)}+c d_{J}^{-1 / 2}\left\|y-y_{h}\right\|_{L^{2}\left(\Omega_{J}^{\prime}\right)} .
$$

This is essentially Corollary 5.1 of [28] where the authors have already inserted an interpolation error estimate. In Example 10.1 of 32 the author proved that this result is also applicable for the domains $\Omega_{J}^{\prime}$, i.e., for domains which abut on the boundary but contain no corner point. Let $\delta \in[0,1 / 2]$. Using (3.29) and (3.30) we arrive at

$$
\begin{aligned}
& \left\|y-y_{h}\right\|_{L^{2}\left(\Gamma_{R / 16}^{ \pm}\right)}=\left(\sum_{J=4}^{I}\left\|y-y_{h}\right\|_{L^{2}\left(\partial \Omega_{J}^{ \pm}\right)}^{2}\right)^{1 / 2} \\
& \leq c\left(\sum_{J=4}^{I}\left(d_{J}^{1 / 2}|\ln h|\left\|y-I_{h} y\right\|_{L^{\infty}\left(\Omega_{J}^{\prime}\right)}+d_{J}^{-1 / 2}\left\|y-y_{h}\right\|_{L^{2}\left(\Omega_{J}^{\prime}\right)}\right)^{2}\right)^{1 / 2} \\
& \leq c|\ln h| \max _{4 \leq J \leq I}\left(d_{J}^{\delta}\left\|y-I_{h} y\right\|_{L^{\infty}\left(\Omega_{J}^{\prime}\right)}\right)\left(\sum_{J=4}^{I} d_{J}^{1-2 \delta}\right)^{1 / 2}+c\left(\sum_{J=4}^{I}\left\|d_{J}^{-1 / 2}\left(y-y_{h}\right)\right\|_{L^{2}\left(\Omega_{J}^{\prime}\right)}^{2}\right)^{1 / 2} .
\end{aligned}
$$

An application of the discrete Hölder inequality yields

$$
\left(\sum_{J=4}^{I} d_{J}^{1-2 \delta}\right)^{1 / 2} \leq\left(\sum_{J=4}^{I} d_{J}\right)^{(1-2 \delta) / 2}\left(\sum_{J=4}^{I} 1\right)^{\delta} \leq c|\ln h|^{\delta}
$$


where we have used $\sum_{J=4}^{I} d_{J} \sim\left|\Gamma_{R / 16}^{ \pm}\right|$and $\sum_{J=4}^{I} 1 \sim|\ln h|$ in the last step. Thus, we obtain

$$
\begin{aligned}
\left\|y-y_{h}\right\|_{L^{2}\left(\Gamma_{R / 16}^{ \pm}\right)} & \leq c|\ln h|^{1+\delta} \max _{4 \leq J \leq I}\left(d_{J}^{\delta}\left\|y-I_{h} y\right\|_{L^{\infty}\left(\Omega_{J}^{\prime}\right)}\right) \\
& +c\left\|\left(r+d_{I}\right)^{-1 / 2}\left(y-y_{h}\right)\right\|_{L^{2}\left(\Omega_{R / 8}\right)} .
\end{aligned}
$$

Let $1<q \leq \infty,-2 / q<\gamma<2-2 / q$ and $\gamma \leq 2+\delta-2 \mu \leq 5 / 2-2 \mu$. Then we get with Lemma 3.7 and Lemma 3.10.

$$
\begin{aligned}
\left\|y-y_{h}\right\|_{L^{2}\left(\Gamma_{R / 16}^{ \pm}\right)} & \leq c h^{2}|\ln h|^{1+\delta} \max _{4 \leq J \leq I}|y|_{W_{\gamma}^{2, \infty}\left(\Omega_{J}^{\prime \prime}\right)} \\
& +c\left(h^{2}|\ln h|^{1 / 2}|y|_{W_{\gamma}^{2, \infty}\left(\Omega_{R}\right)}+\left\|y-y_{h}\right\|_{L^{2}\left(\Omega_{R}\right)}\right),
\end{aligned}
$$

which ends the proof.

Now we are able to prove Theorem 3.2 .

Proof. We split the error on the boundary into the already introduced boundary parts,

$$
\left\|y-y_{h}\right\|_{L^{2}(\Gamma)} \leq c\left(\sum_{j=1}^{m}\left\|y-y_{h}\right\|_{L^{2}\left(\Gamma_{R_{j} / 16}^{ \pm}\right)}+\left\|y-y_{h}\right\|_{L^{2}\left(\tilde{\Gamma}^{0}\right)}\right) .
$$

For each boundary part $\Gamma_{R_{j} / 16}^{ \pm}, j=1, \ldots, m$, we get from Lemma 3.12

$$
\left\|y-y_{h}\right\|_{L^{2}\left(\Gamma_{R_{j} / 16}^{ \pm}\right)} \leq c\left(h^{2}|\ln h|^{1+\delta}|y|_{W_{\gamma_{j}}^{2, \infty}\left(\Omega_{R_{j}}\right)}+\left\|y-y_{h}\right\|_{L^{2}\left(\Omega_{R_{j}}\right)}\right),
$$

provided that $0 \leq \delta \leq 1 / 2,1<q_{j} \leq \infty, \gamma_{j} \leq 2+\delta-2 \mu_{j}$ and $-2 / q_{j}<\gamma_{j}<2-2 / q_{j}$. If we set $\mu_{j} \geq \delta / 2$ we can choose $q_{j}$ such that $1 \leq 2 /\left(2 \mu_{j}-\delta\right)<q_{j}<\infty$. By this we get that (3.32) is valid for $0 \leq \gamma_{j} \leq 2+\delta-2 \mu_{j}$ with some arbitrary $\delta \in[0,1 / 2]$, i.e., it holds that

$$
\left\|y-y_{h}\right\|_{L^{2}\left(\Gamma_{R_{j} / 16}^{ \pm}\right)} \leq c\left(h^{2}|\ln h|^{1+\delta}|y|_{W_{2+\delta-2 \mu_{j}}^{2, \infty}\left(\Omega_{R_{j}}\right)}+\left\|y-y_{h}\right\|_{L^{2}\left(\Omega_{R_{j}}\right)}\right)
$$

for every $\delta \in[0,1 / 2]$. Next, we estimate the last term in the right-hand side of (3.31). We can conclude from the embedding $L^{\infty}\left(\Gamma^{0}\right) \hookrightarrow L^{2}\left(\Gamma^{0}\right)$ and the fact that $y-y_{h}$ is a continuous function on $\operatorname{cl}\left(\tilde{\Omega}^{0}\right)$ that

$$
\left\|y-y_{h}\right\|_{L^{2}\left(\tilde{\Gamma}^{0}\right)} \leq c\left\|y-y_{h}\right\|_{L^{\infty}\left(\tilde{\Gamma}^{0}\right)} \leq c\left\|y-y_{h}\right\|_{L^{\infty}\left(\tilde{\Omega}^{0}\right)} .
$$

Next we use Theorem 10.1 in 32 with $s=0$ to get

$$
\left\|y-y_{h}\right\|_{L^{2}\left(\tilde{\Gamma}^{0}\right)} \leq c\left(|\ln h|\left\|y-I_{h} y\right\|_{L^{\infty}\left(\check{\Omega}^{0}\right)}+\left\|y-y_{h}\right\|_{L^{2}\left(\check{\Omega}^{0}\right)}\right),
$$

Compare the proof of Lemma 3.12 for the applicability of this theorem in that case. Since the domain $\check{\Omega}^{0} \subset \Omega^{0}$ has a constant, positive distance to the corner, we can conclude using standard interpolation theory that

$$
\left\|y-y_{h}\right\|_{L^{2}\left(\tilde{\Gamma}^{0}\right)} \leq c\left(h^{2}|\ln h||y|_{W^{2, \infty}\left(\Omega^{0}\right)}+\left\|y-y_{h}\right\|_{L^{2}\left(\check{\Omega}^{0}\right)}\right) .
$$

Combining the inequalities (3.31), (3.33) and (3.34) we obtain

$$
\left\|y-y_{h}\right\|_{L^{2}(\Gamma)} \leq c\left(h^{2}|\ln h|^{1+\delta}|y|_{W_{\overrightarrow{2}+\vec{\delta}-2 \vec{\mu}}^{2, \infty}(\Omega)}+\left\|y-y_{h}\right\|_{L^{2}(\Omega)}\right) .
$$


Using Lemma 2.4 we can conclude for some arbitrary $\sigma \in(0,1)$,

$$
\|y\|_{W_{\overrightarrow{2}+\vec{\delta}-2 \vec{\mu}}^{2, \infty}(\Omega)} \leq c\|f\|_{C^{0, \sigma}(\bar{\Omega})},
$$

if $\mu_{j}>\delta / 2$ and $\max \left(0,2-\lambda_{j}\right)<2+\delta-2 \mu_{j}$ or $0 \leq 2+\delta-2 \mu_{j}$ in the case that $2-\lambda_{j}<0$. The latter condition is fulfilled for any $\mu_{j} \in(0,1]$. The former one is equivalent to $\mu_{j}<\delta / 2+\lambda_{j} / 2$ if $\mu_{j} \in(\delta / 2,1]$. It follows from Theorem 3.1 that

$$
\left\|y-y_{h}\right\|_{L^{2}(\Omega)} \leq c h^{2}\|f\|_{W_{\overrightarrow{1}-\vec{\mu}}^{0,2}(\Omega)} \leq c h^{2}\|f\|_{L^{2}(\Omega)},
$$

if $\mu_{j}<\lambda_{j}$. Finally, the inequalities (3.35), (3.36) and (3.37) yield together with the embedding $C^{0, \sigma}(\bar{\Omega}) \hookrightarrow L^{2}(\Omega)$ the desired result.

\section{The CONTINuOUS OPTIMAL CONTROL PROBLEM}

In this section we state the continuous optimality system for problem (1.2) and describe the regularity of its solution in weighted Sobolev spaces. The exposition follows those in [1,20. The solution operator $S: L^{2}(\Gamma) \rightarrow L^{2}(\Omega)$ which associates a state $y=S u$ to a control $u$ via (1.3) has already been introduced in Section [1] We denote with $S^{*}: L^{2}(\Omega) \rightarrow L^{2}(\Gamma)$ the adjoint operator of $S$. One has

$$
(S u, z)_{L^{2}(\Omega)}=\left(u, S^{*} z\right)_{L^{2}(\Gamma)} \quad \forall u \in L^{2}(\Gamma), z \in L^{2}(\Omega) .
$$

Furthermore, we define the operator $P: L^{2}(\Omega) \rightarrow H^{1}(\Omega)$ by $P z:=p$ where $p$ is the solution of

$$
\begin{aligned}
-\Delta p+p=z & \text { in } \Omega, \\
\partial_{n} p=0 & \text { on } \Gamma .
\end{aligned}
$$

The operators $S^{*}$ and $P$ are related by $S^{*} z=(P z)_{\mid \Gamma}=p_{\mid \Gamma}$. We can also associate an adjoint state to every control $u$ by $P\left(S u-y_{d}\right)$. Finally, we define the projection

$$
\Pi_{[a, b]} f(x):=\max (a, \min (b, f(x)))
$$

and the set of admissible controls as

$$
U_{a d}:=\left\{u \in L^{2}(\Gamma): a \leq u \leq b \text { a.e. on } \Gamma\right\} .
$$

Theorem 4.1. The optimal control problem (1.2) has a unique solution $\bar{u} \in$ $L^{2}(\Gamma)$. Let $\bar{y}=S \bar{u}$ and $\bar{p}=P\left(S \bar{u}-y_{d}\right)$ be the state and adjoint state associated with $\bar{u}$. Then the variational inequality

$$
(\bar{p}+\nu \bar{u}, u-\bar{u})_{L^{2}(\Gamma)} \geq 0 \quad \forall u \in U_{a d}
$$

is satisfied, which can be expressed equivalently by

$$
\bar{u}(x)=\Pi_{[a, b]}\left(-\frac{1}{\nu} \bar{p}(x)\right) \quad \text { for a. } a . x \in \Gamma .
$$

Moreover, let $y_{d} \in C^{0, \sigma}(\bar{\Omega})$ with some $\sigma \in(0,1)$ and let $\beta_{j}$ and $\gamma_{j}$ satisfy the conditions

$$
\begin{aligned}
& 1>\beta_{j}>\max \left(0,1-\lambda_{j}\right) \quad \text { or } \quad \beta_{j}=0 \text { and } 1-\lambda_{j}<0, \\
& 2>\gamma_{j}>\max \left(0,2-\lambda_{j}\right) \quad \text { or } \quad \gamma_{j}=0 \text { and } 2-\lambda_{j}<0,
\end{aligned}
$$

for $j=1, \ldots, m$. Then $\bar{y}$ belongs to $W_{\vec{\beta}}^{2,2}(\Omega), \bar{p}$ to $W_{\vec{\gamma}}^{2, \infty}(\Omega)$ and $\bar{p}_{\mid \Gamma}$ to $W_{\vec{\gamma}}^{2, \infty}(\Gamma)$.

Furthermore, the following estimate is valid:

$$
\|\bar{y}\|_{W_{\vec{\beta}}^{2,2}(\Omega)}+\|\bar{p}\|_{W_{\vec{\gamma}}^{2, \infty}(\Omega)}+\|\bar{p}\|_{W_{\vec{\gamma}}^{2, \infty}(\Gamma)} \leq c\left(\|\bar{u}\|_{L^{2}(\Gamma)}+\left\|y_{d}\right\|_{C^{0, \sigma}(\bar{\Omega})}\right) .
$$


Proof. Since the optimal control problem is linear quadratic and strictly convex, the existence and the uniqueness of a solution $\bar{u} \in L^{2}(\Gamma)$ is an immediate result; compare also [6, 7, 20, The variational inequality (4.2) represents the necessary optimality condition which is also sufficient due to the strict convexity. The proof of the equivalence between the variational inequality (4.2) and the projection formula (4.3) can be found, e.g., in [7]. To prove the assertion on the regularity and the a priori estimates we start with the optimal control $\bar{u}$ in $L^{2}(\Gamma)$. This implies according to [15] or Theorem 2.1 in [7] that $\bar{y}$ belongs to $H^{3 / 2}(\Omega)$ and

$$
\|\bar{y}\|_{H^{3 / 2}(\Omega)} \leq c\|\bar{u}\|_{L^{2}(\Gamma)} .
$$

Furthermore, one can conclude for any $\epsilon \in(0,1 / 2] \cap(0, \sigma]$ with the Sobolev embedding theorem that

$$
\|\bar{y}\|_{C^{0, \epsilon}(\bar{\Omega})} \leq c\|\bar{y}\|_{H^{3 / 2}(\Omega)} .
$$

Based on this, using the results of Lemma 2.4, we get that $\bar{p}$ admits the estimate

$$
\|\bar{p}\|_{W_{\vec{\gamma}}^{2, \infty}(\Omega)}+\|\bar{p}\|_{W_{\vec{\gamma}}^{2, \infty}(\Gamma)} \leq c\left\|y-y_{d}\right\|_{C^{0, \epsilon}(\bar{\Omega})} \leq c\left(\|y\|_{C^{0, \epsilon}(\bar{\Omega})}+\left\|y_{d}\right\|_{C^{0, \epsilon}(\bar{\Omega})}\right),
$$

provided that condition (4.5) is satisfied. Due to the Lax-Milgram Theorem and a standard trace theorem one also gets that

$$
\|\bar{p}\|_{H^{1 / 2}(\Gamma)} \leq c\|\bar{p}\|_{H^{1}(\Omega)} \leq c\left\|y-y_{d}\right\|_{L^{2}(\Omega)} \leq c\left(\|y\|_{C^{0, \epsilon}(\bar{\Omega})}+\left\|y_{d}\right\|_{C^{0, \epsilon}(\bar{\Omega})}\right) .
$$

Since the optimal control $\bar{u}$ is related to the optimal adjoint state $\bar{p}$ via the projection formula (4.3) we obtain $\bar{u} \in H^{1 / 2}(\Gamma)$ and

$$
\begin{aligned}
\|\bar{u}\|_{H^{1 / 2}(\Gamma)} & \leq\|\bar{u}\|_{L^{2}(\Gamma)}+\left(\int_{\Gamma} \int_{\Gamma} \frac{\left|\bar{u}\left(x_{1}\right)-\bar{u}\left(x_{2}\right)\right|^{2}}{\left|x_{1}-x_{2}\right|^{2}} \mathrm{~d} s_{x_{1}} \mathrm{~d} s_{x_{2}}\right)^{1 / 2} \\
& =\|\bar{u}\|_{L^{2}(\Gamma)}+\left(\int_{\Gamma} \int_{\Gamma} \frac{\left|\Pi_{[a, b]}\left(-\frac{1}{\nu} \bar{p}\left(x_{1}\right)\right)-\Pi_{[a, b]}\left(-\frac{1}{\nu} \bar{p}\left(x_{2}\right)\right)\right|^{2}}{\left|x_{1}-x_{2}\right|^{2}} \mathrm{~d} s_{x_{1}} \mathrm{~d} s_{x_{2}}\right)^{1 / 2} \\
& \leq\|\bar{u}\|_{L^{2}(\Gamma)}+c\left(\int_{\Gamma} \int_{\Gamma} \frac{\left|\bar{p}\left(x_{1}\right)-\bar{p}\left(x_{2}\right)\right|^{2}}{\left|x_{1}-x_{2}\right|^{2}} \mathrm{~d} s_{x_{1}} \mathrm{~d} s_{x_{2}}\right)^{1 / 2} .
\end{aligned}
$$

The last step can easily be verified, if one distinguishes the nine cases $-\bar{p}\left(x_{1}\right) / \nu<$ $a \wedge-\bar{p}\left(x_{2}\right) / \nu<a,-\bar{p}\left(x_{1}\right) / \nu<a \wedge a \leq-\bar{p}\left(x_{2}\right) / \nu \leq b,-\bar{p}\left(x_{1}\right) / \nu<a \wedge$ $-\bar{p}\left(x_{2}\right) / \nu>b, a \leq-\bar{p}\left(x_{1}\right) / \nu \leq b \wedge-\bar{p}\left(x_{2}\right) / \nu<a$, etc. Using the embedding $H^{1 / 2}(\Gamma) \hookrightarrow W_{\vec{\beta}}^{1 / 2,2}(\Gamma)$, which is valid for $\vec{\beta} \geq \overrightarrow{0}$, yields together with Lemma 2.3 ,

$$
\|\bar{y}\|_{W_{\vec{\beta}}^{2,2}(\Omega)} \leq c\|\bar{u}\|_{W_{\vec{\beta}}^{1 / 2,2}(\Gamma)} \leq c\|\bar{u}\|_{H^{1 / 2}(\Gamma)},
$$

provided that the condition (4.4) is fulfilled. Finally, the estimates (4.6), (4.7), (4.8), (4.9), (4.10) and (4.11) yield the desired result.

Corollary 4.2. Let $y_{d} \in C^{0, \sigma}(\bar{\Omega})$ with some $\sigma \in(0,1)$ and let $\beta_{j}$ satisfy the condition

$$
1 / 2>\beta_{j}>\max \left(0,3 / 4-\lambda_{j} / 2\right) \quad \text { or } \quad \beta_{j}=0 \text { and } 3 / 4-\lambda_{j} / 2<0
$$

for $j=1, \ldots, m$. Then the optimal adjoint state $\left.\bar{p}\right|_{\Gamma}=S^{*}\left(S \bar{u}-y_{d}\right)$ belongs to $W_{2 \vec{\beta}}^{2,2}(\Gamma) \hookrightarrow W_{\vec{\beta}}^{1, \infty}(\Gamma)$ and the following a priori estimate holds:

$$
\|\bar{p}\|_{W_{\vec{\beta}}^{1, \infty}(\Gamma)} \leq c\|\bar{p}\|_{W_{2 \vec{\beta}}^{2,2}(\Gamma)} \leq c\left(\|\bar{u}\|_{L^{2}(\Gamma)}+\left\|y_{d}\right\|_{C^{0, \sigma}(\bar{\Omega})}\right) .
$$


Proof. We begin by proving that the first inequality of (4.13) holds for $0 \leq \beta_{j}<$ $1 / 2, j=1, \ldots, m$. The Sobolev inequality yields, with some $q \in(1,2]$,

$$
\begin{aligned}
\|\bar{p}\|_{W_{\vec{\beta}}^{1, \infty}(\Gamma)} & =\|\bar{p}\|_{W^{1, \infty}\left(\Gamma^{0}\right)}+\sum_{j=1}^{m} \sum_{|\alpha| \leq 1}\left\|r_{j}^{\beta_{j}} \partial_{t}^{\alpha} \bar{p}\right\|_{L^{\infty}\left(\Gamma_{j}^{ \pm}\right)} \\
& \leq\|\bar{p}\|_{W^{2,2}\left(\Gamma^{0}\right)}+c \sum_{j=1}^{m} \sum_{|\alpha| \leq 1}\left\|r_{j}^{\beta_{j}} \partial_{t}^{\alpha} \bar{p}\right\|_{W^{1, q}\left(\Gamma_{j}^{ \pm}\right)}
\end{aligned}
$$

For $0<\beta_{j}<1 / 2$ we set $1 / q=\max ((\overrightarrow{2}-\vec{\beta}) / 2,(\overrightarrow{3}+2 \vec{\beta}) / 4)$. Thus, $1<q<2$. We get with the product rule and part 1 of Lemma 2.1 that

$$
\begin{aligned}
& \sum_{|\alpha| \leq 1}\left\|r_{j}^{\beta_{j}} \partial_{t}^{\alpha} \bar{p}\right\|_{W^{1, q}\left(\Gamma_{j}^{ \pm}\right)} \\
& \quad \leq c\left(\|\bar{p}\|_{W_{\beta_{j}-1}^{0, q}\left(\Gamma_{j}^{ \pm}\right)}+\|\bar{p}\|_{W_{\beta_{j}-1}^{1, q}\left(\Gamma_{j}^{ \pm}\right)}+\|\bar{p}\|_{W_{\beta_{j}}^{2, q}\left(\Gamma_{j}^{ \pm}\right)}\right) \\
& \quad \leq c\|\bar{p}\|_{W_{\beta_{j}}^{2, q}\left(\Gamma_{j}^{ \pm}\right)} \leq c\|\bar{p}\|_{W_{2 \beta_{j}}^{2,2}\left(\Gamma_{j}^{ \pm}\right)} .
\end{aligned}
$$

In the case that $\beta_{j}=0$ we directly get this result from (4.14) with $q=2$. In summary, one obtains from the inequalities (4.14) and (4.15) for $0 \leq \beta_{j}<1 / 2$ the validity of the first inequality of (4.13). Finally, if we set the weights $\beta_{j}$ according to (4.12) and define the weights $\delta_{j}=\max \left(0,7 / 8+3 \beta_{j} / 2-\lambda_{j} / 4\right)$ for $j=1, \ldots, m$ in a clever way, then we get using part 2 of Lemma 2.1 and Theorem 4.1 the desired result

$$
\|\bar{p}\|_{W_{2 \bar{\beta}}^{2,2}(\Gamma)} \leq c\|\bar{p}\|_{W_{\vec{\delta}}^{2, \infty}(\Gamma)} \leq c\left(\|\bar{u}\|_{L^{2}(\Gamma)}+\left\|y_{d}\right\|_{C^{0, \sigma}(\bar{\Omega})}\right)
$$

with some $\sigma \in(0,1)$.

\section{The Discrete optimal CONTRol PROBlem}

In this section we fully discretize the optimal control problem (1.2). The state and the adjoint state will be discretized by piecewise linear and globally continuous functions. The control will be approximated by piecewise constant functions.

In Section 3 we have already introduced the graded triangulations $\mathcal{T}_{h}$ of $\Omega$ with its boundary triangulations $\mathcal{E}_{h}$. The space $V_{h}$ has been defined by

$$
V_{h}=\left\{y_{h} \in C(\bar{\Omega}):\left.y_{h}\right|_{T} \in \mathcal{P}_{1}(T) \forall T \in \mathcal{T}_{h}\right\} .
$$

Furthermore, we introduce the spaces

$$
U_{h}:=\left\{u_{h} \in L^{\infty}(\Gamma):\left.u_{h}\right|_{G} \in \mathcal{P}_{0}(G) \forall G \in \mathcal{E}_{h}\right\}
$$

and

$$
U_{h}^{a d}:=U_{h} \cap U_{a d}
$$

The discrete variant of the state equation reads as follows: Find for each $u \in L^{2}(\Gamma)$ the unique element $y_{h} \in V_{h}$ satisfying

$$
a\left(y_{h}, v_{h}\right)=\left(u, v_{h}\right)_{L^{2}(\Gamma)} \quad \forall v_{h} \in V_{h},
$$

where the bilinear form $a: H^{1}(\Omega) \times H^{1}(\Omega) \rightarrow \mathbb{R}$ is defined in (2.4). We denote with $S_{h}: L^{2}(\Gamma) \rightarrow L^{2}(\Omega)$ the discrete solution operator which maps a control $u$ to 
$S_{h} u:=y_{h}$ via (5.1). The fully discretized version of the optimal control problem can now be stated as

$$
J_{h}\left(\bar{u}_{h}\right)=\min _{u_{h} \in U_{h}^{a d}} J_{h}\left(u_{h}\right)
$$

with

$$
J_{h}\left(u_{h}\right):=\frac{1}{2}\left\|S_{h} u_{h}-y_{d}\right\|_{L^{2}(\Omega)}^{2}+\frac{\nu}{2}\left\|u_{h}\right\|_{L^{2}(\Gamma)} \cdot
$$

As in the continuous case we can deduce an optimality system for the discrete problem. For that purpose we also introduce the discrete version of the solution operator $P$ which is denoted by $P_{h}: L^{2}(\Omega) \rightarrow H^{1}(\Omega)$ and defined by $P_{h} z=p_{h}$ with some function $z \in L^{2}(\Omega)$ and $p_{h}$ being the unique element in $V_{h}$ such that

$$
a\left(v_{h}, p_{h}\right)=\left(z, v_{h}\right)_{L^{2}(\Omega)} \quad \forall v_{h} \in V_{h} .
$$

Let $S_{h}^{*}: L^{2}(\Omega) \rightarrow L^{2}(\Gamma)$ denote the discretized version of the operator $S^{*}$. Then we can conclude that $S_{h}^{*} z=\left(P_{h} z\right)_{\mid \Gamma}$, since the adjoint of the discrete solution operator is equal to the discretization of the adjoint solution operator. Furthermore, we have that

$$
\left(S_{h} u, z\right)_{L^{2}(\Omega)}=\left(u, S_{h}^{*} z\right)_{L^{2}(\Gamma)} \quad \forall z \in L^{2}(\Omega), \forall u \in L^{2}(\Gamma) .
$$

Finally, the discrete adjoint state is the unique element $P_{h}\left(S_{h} u-y_{d}\right) \in V_{h}$.

Lemma 5.1. The discrete optimal control problem (5.2) admits a unique solution $\bar{u}_{h}$. Let $\bar{y}_{h}=S_{h} \bar{u}_{h}$ and $\bar{p}_{h}=P_{h}\left(S_{h} \bar{u}_{h}-y_{d}\right)$ be the discrete state and discrete adjoint state associated with $\bar{u}_{h}$. Then the discrete variational inequality

$$
\left(\bar{p}_{h}+\nu \bar{u}_{h}, u_{h}-\bar{u}_{h}\right)_{L^{2}(\Gamma)} \geq 0 \quad \forall u_{h} \in U_{a d}^{h}
$$

is satisfied.

Proof. This follows analogously to the continuous case.

\section{RESUlTS FROM NUMERICAL INTEGRATION}

For the subsequent discretization error analysis of the optimal control problem we need some results from numerical integration on the boundary. Remember, that the triangulation $\mathcal{T}_{h}$ of the domain $\Omega$ induces a segmentation $\mathcal{E}_{h}$ of the boundary $\Gamma$. We define the distance of the edge $E$ to the corner $x^{(j)}$ by $r_{E, j}:=\inf _{x \in E}\left|x-x^{(j)}\right|$ and the element size $h_{E}$ by $h_{E}:=\operatorname{diam} E$. According to (3.1) there holds

$$
\begin{aligned}
c_{1} h^{1 / \mu_{j}} & \leq h_{E} \leq c_{2} h^{1 / \mu_{j}} & & \text { for } r_{E, j}=0, \\
c_{1} h r_{E, j}^{1-\mu_{j}} & \leq h_{E} \leq c_{2} h r_{E, j}^{1-\mu_{j}} & & \text { for } 0<r_{E, j} \leq R_{j}, \\
c_{1} h & \leq h_{E} \leq c_{2} h & & \text { for } r_{E, j}>R_{j},
\end{aligned}
$$

for $j=1, \ldots, m$. Furthermore, for $j=1, \ldots, m$ let $\mathcal{E}_{h, j}$ be the subtriangulation of $\mathcal{E}_{h}$ such that $\bigcup_{E \in \mathcal{E}_{h, j}} \bar{E} \subset \Gamma_{j}^{ \pm}$and $E \cap \Gamma_{j}^{ \pm} \neq E$ for all $E \notin \mathcal{E}_{h, j}$. We set $\mathcal{E}_{h, 0}=\mathcal{E}_{h} \backslash \bigcup_{j=1}^{n} \mathcal{E}_{h, j}$.

Now, let $S_{E}$ be the midpoint of the edge $E \in \mathcal{E}_{h}$. Then the projection operator $R_{h}$ is defined as the 0 -interpolator onto $U_{h}$, i.e.,

$$
R_{h}: C(\Gamma) \rightarrow U_{h}, f \rightarrow R_{h} f,
$$

where

$$
\left(R_{h} f\right)(x):=f\left(S_{E}\right) \quad \text { if } x \in E .
$$


In the proof of Lemma 3.7 we have already derived interpolation error estimates in $T$. There we used the embedding $W^{2, q^{\prime}}(\hat{T}) \hookrightarrow L^{\infty}(\hat{T})$ if $q^{\prime}>1$. Afterwards we applied the Deny-Lions Lemma and embeddings, which hold for weighted Sobolev spaces (cf. the steps between (3.13) and (3.16)). If we would do the same for boundary elements $E$, we would get a condition that is too restrictive on the mesh grading parameters $\mu_{j}$. For that reason we use Lemma 2.2 in the proof of the following lemma about the approximation properties of the operator $R_{h}$.

Lemma 6.1. Let $1 / 4<\mu_{j} \leq 1$. Then the estimate

$$
\left|\int_{E}\left(f-R_{h} f\right) d s\right| \leq c h^{2}|E|^{1 / 2}|f|_{W_{2\left(1-\mu_{j}\right)}^{2,2}(E)}
$$

holds true for any element $E \in \mathcal{E}_{h, j}$ and every function $f \in W_{2\left(1-\mu_{j}\right)}^{2,2}(E)$.

Proof. First, we observe that the integral vanishes for any polynomial $p$ of order one, hence

$$
\begin{aligned}
\left|\int_{E}\left(f-R_{h} f\right) d s\right| & =\left|\int_{E}\left(f-p-R_{h}(f-p)\right) d s\right| \\
& \leq|E|\left(\|f-p\|_{L^{\infty}(E)}+\left\|R_{h}(f-p)\right\|_{L^{\infty}(E)}\right) \\
& \leq c|E|\|f-p\|_{L^{\infty}(E)} .
\end{aligned}
$$

In the last step we used that $R_{h}$ is a bounded operator from $L^{\infty}(E)$ to $L^{\infty}(E)$ with $\left\|R_{h}\right\|_{L^{\infty}(E) \rightarrow L^{\infty}(E)}=1$. Now we distinguish between edges $E$ with $r_{E, j}>0$ and $r_{E, j}=0$. In the first case we use the embedding $W^{2,2}(\hat{E}) \hookrightarrow L^{\infty}(\hat{E})$ and the Deny-Lions Lemma [10] after the transformation to the reference edge $\hat{E}$. We obtain

$$
\|f-p\|_{L^{\infty}(E)}=\|\hat{f}-\hat{p}\|_{L^{\infty}(\hat{E})} \leq c\|\hat{f}-\hat{p}\|_{W^{2,2}(\hat{E})} \leq c|\hat{f}|_{W^{2,2}(\hat{E})} .
$$

The reverse transformation together with $h_{E} \sim h r_{E, j}^{1-\mu}$ yields

$$
\left|\int_{E}\left(f-R_{h} f\right) d s\right| \leq c|E|^{1 / 2} h_{E}^{2}|f|_{W^{2,2}(E)} \leq c|E|^{1 / 2} h^{2}|f|_{W_{2\left(1-\mu_{j}\right)}^{2,2}(E)} .
$$

In the second case, $r_{E, j}=0$, we use the embedding $W_{2\left(1-\mu_{j}\right)}^{2,2}(\hat{E}) \hookrightarrow W_{1}^{2,4 /\left(5-4 \mu_{j}\right)}(\hat{E})$ $\hookrightarrow W^{1,4 /\left(5-4 \mu_{j}\right)}(\hat{E}) \hookrightarrow L^{\infty}(\hat{E})$ (see Section 9), which holds for $1 / 4<\mu_{j} \leq 1$ (cf. parts 2 and 1 Lemma 2.1). By this we obtain, using Lemma 2.2

$$
\begin{aligned}
\|f-p\|_{L^{\infty}(E)} & =\|\hat{f}-\hat{p}\|_{L^{\infty}(\hat{E})} \leq c\|\hat{f}-\hat{p}\|_{W_{2\left(1-\mu_{j}\right)}^{2,2}(\hat{E})} \\
& \leq c\left(|\hat{f}-\hat{p}|_{W_{2\left(1-\mu_{j}\right)}^{2,2}(\hat{E})}+\sum_{\delta \leq 1}\left|\int_{\hat{E}} \partial_{t}^{\delta}(\hat{f}-\hat{p}) d \hat{s}\right|\right) \\
& =c\left(|\hat{f}|_{W_{2\left(1-\mu_{j}\right)}^{2,2}(\hat{E})}+\sum_{\delta \leq 1}\left|\int_{\hat{E}} \partial_{t}^{\delta}(\hat{f}-\hat{p}) d \hat{s}\right|\right),
\end{aligned}
$$

since $p$ is polynomial of order one. Next, we choose $p$ such that the last term of (6.4) vanishes, which is possible without any restriction. This yields

$$
\|f-p\|_{L^{\infty}(E)} \leq c|\hat{f}|_{W_{2\left(1-\mu_{j}\right)}^{2,2}(\hat{E})} \leq c h_{E}^{2 \mu_{j}}|E|^{-1 / 2}|f|_{W_{2\left(1-\mu_{j}\right)}^{2,2}(E)},
$$


where we used $\hat{r}_{j}^{2\left(1-\mu_{j}\right)} \sim h_{E}^{-2\left(1-\mu_{j}\right)} r_{j}^{2\left(1-\mu_{j}\right)}$. The inequalities (6.2) and (6.5) yield with $h_{E} \sim h^{1 / \mu_{j}}$ the assertion for $r_{E, j}=0$.

Corollary 6.2. Let $E \in \mathcal{E}_{h}$ and $f \in W^{2,2}(E)$. Then the estimate

$$
\left|\int_{E}\left(f-R_{h} f\right) d s\right| \leq c h^{2}|E|^{1 / 2}|f|_{W^{2,2}(E)}
$$

is valid.

Proof. This follows from (6.2) and (6.3) together with $h_{E} \leq$ ch after the reverse transformation to the edge $E$.

Lemma 6.3. Let $0<\mu_{j} \leq 1$. Then the estimate

$$
\left|\int_{E}\left(f-R_{h} f\right) d s\right| \leq c h|E||f|_{W_{1-\mu_{j}}^{1, \infty}(E)}
$$

holds true for any element $E \in \mathcal{E}_{h, j}$ and every function $f \in W_{1-\mu_{j}}^{1, \infty}(E)$.

Proof. We get from (6.2),

$$
\begin{aligned}
\left|\int_{E}\left(f-R_{h} f\right) d s\right| & \leq c|E|\|f-p\|_{L^{\infty}(E)} \leq c|E|\|\hat{f}-\hat{p}\|_{L^{\infty}(\hat{E})} \\
& \leq c|E|\|\hat{f}-\hat{p}\|_{W^{1, q}(\hat{E})} \leq c|E \| \hat{f}|_{W^{1, q}(\hat{E})}
\end{aligned}
$$

where we used the embedding $W^{1, q}(\hat{E}) \hookrightarrow L^{\infty}(\hat{E})$ with some $q>1$ and the DenyLions Lemma [10]. Now we consider the case that $r_{E, j}>0$. After the reverse transformation to the edge $E$ one gets with $h_{E} \sim h r_{E, j}^{1-\mu_{j}}$,

$\left|\int_{E}\left(f-R_{h} f\right) d s\right| \leq c|E|^{1-1 / q} h_{E}|f|_{W^{1, q}(E)} \leq c|E| h r_{E, j}^{1-\mu_{j}}|f|_{W^{1, \infty}(E)} \leq c|E| h|f|_{W_{1-\mu_{j}}^{1, \infty}(E)}$.

In the case that $r_{E, j}=0$ we can conclude using (6.6) with $q=2 /\left(2-\mu_{j}\right)>1$ and the embedding $W_{1-\mu_{j}}^{1, \infty}(\hat{E}) \hookrightarrow W^{1,2 /\left(2-\mu_{j}\right)}(\hat{E})$, which holds for $0<\mu_{j} \leq 1$,

$$
\left|\int_{E}\left(f-R_{h} f\right) d s\right| \leq c|E||\hat{f}|_{W_{1-\mu_{j}}^{1, \infty}(\hat{E})} .
$$

Finally, the reverse transformation to the edge $E$ together with $\hat{r}_{j}^{1-\mu_{j}} \sim h_{E}^{\mu_{j}-1} r_{j}^{1-\mu_{j}}$ and $h_{E} \sim h^{1 / \mu_{j}}$ yields

$$
\left|\int_{E}\left(f-R_{h} f\right) d s\right| \leq c|E| h_{E} h_{E}^{\mu_{j}-1}|f|_{W_{1-\mu_{j}}^{1, \infty}(E)} \leq c|E| h|f|_{W_{1-\mu_{j}}^{1, \infty}(E)} .
$$

Corollary 6.4. Let $E \in \mathcal{E}_{h}$ and $f \in W^{1, \infty}(E)$. Then the estimate

$$
\left|\int_{E}\left(f-R_{h} f\right) d s\right| \leq c h|E||f|_{W^{1, \infty}(E)}
$$

holds.

Proof. One gets this result from (6.6) with $q=\infty$ and $h_{E} \leq$ ch after the reverse transformation to the edge $E$. 


\section{Discretization error estimates for the optimal CONTRol Problem}

Based on the results of the previous sections we first analyze in the following the fully discrete optimal control problem of Section 5 with respect to its discretization error. Afterwards we construct in a postprocessing step a new control which possesses better approximation properties. Let

$$
K_{1}:=\bigcup_{E \in \mathcal{E}_{h}: \bar{u} \notin W_{2(\overrightarrow{1}-\vec{\mu})}^{2,2}(E)} E, \quad K_{2}:=\bigcup_{E \in \mathcal{E}_{h}: \bar{u} \in W_{2(\overrightarrow{1}-\vec{\mu})}^{2,2}(E)} E .
$$

Throughout the rest of this paper we make the following assumption on the measure of the set $K_{1}$.

Assumption 7.1. We assume that meas $\left(K_{1}\right)<c h$.

Remark 7.2. This assumption is satisfied in many practical applications. For example it is fulfilled if the optimal control $\bar{u}$ has only a finite number of kinks due to the projection on the interval $[a, b]$. Section 4 in $[20$ contains a more detailed discussion on the validity of this assumption in the case $\vec{\mu}=\overrightarrow{1}$ (quasi-uniform meshes) where $W_{2(\overrightarrow{1}-\vec{\mu})}^{2,2}(E) \equiv W^{2,2}(E)$. For refined meshes, Assumption [7.1] is even slightly weaker.

Let us define the $L^{2}$-projection of a function $f \in L^{2}(\Gamma)$ as the piecewise constant function in $U_{h}$ that fulfills

$$
\left.Q_{h} f\right|_{E} \equiv \frac{1}{|E|} \int_{E} f(x) d s
$$

on any element $E \in \mathcal{E}_{h}$. The following approximation property of the $L^{2}$-projection is proven in Corollary 4.8 of [1].

Corollary 7.3. For any element $E \in \mathcal{E}_{h}$ and any functions $f \in H^{1}(E)$ and $v \in H^{s}(E), s=[0,1]$, the estimate

$$
\left(f-Q_{h} f, v\right)_{L^{2}(E)} \leq \operatorname{ch}_{T}^{s+1}|f|_{H^{1}(E)}|v|_{H^{s}(E)}
$$

is valid.

Lemma 7.4. Let Assumption 7.1 be satisfied. Then the estimate

$$
\left\|S_{h}\left(\bar{u}-R_{h} \bar{u}\right)\right\|_{L^{2}(\Omega)} \leq \operatorname{ch}^{2}\left(\|\bar{u}\|_{L^{2}(\Gamma)}+\left\|y_{d}\right\|_{C^{0, \sigma}(\bar{\Omega})}\right)
$$

is valid, provided that the mesh parameters $\vec{\mu}$ are chosen such that $\overrightarrow{1} / 2<\vec{\mu}<$ $\overrightarrow{1} / 4+\vec{\lambda} / 2$.

Proof. First, we introduce the function $S\left(\bar{u}-R_{h} \bar{u}\right)$ and apply the triangle inequality. This yields

$$
\left\|S_{h}\left(\bar{u}-R_{h} \bar{u}\right)\right\|_{L^{2}(\Omega)} \leq\left\|\left(S_{h}-S\right)\left(\bar{u}-R_{h} \bar{u}\right)\right\|_{L^{2}(\Omega)}+\left\|S\left(\bar{u}-R_{h} \bar{u}\right)\right\|_{L^{2}(\Omega)} .
$$

Applying the Nitsche method together with $\vec{\mu}<\vec{\lambda}$ (cf. the proof of Lemma 4.1 in 11]) we get for the first term in (7.1),

$$
\begin{aligned}
\left\|\left(S_{h}-S\right)\left(\bar{u}-R_{h} \bar{u}\right)\right\|_{L^{2}(\Omega)} & \leq \operatorname{ch}\left\|S\left(\bar{u}-R_{h} \bar{u}\right)\right\|_{H^{1}(\Omega)} \\
& \leq \operatorname{ch}\left\|\bar{u}-R_{h} \bar{u}\right\|_{L^{2}(\Gamma)} \leq \operatorname{ch}^{2}|\bar{u}|_{W_{\overrightarrow{0}}^{1,2}(\Gamma)},
\end{aligned}
$$


where we used the a priori estimate given by the Lax-Milgram Theorem, a standard interpolation error estimate and $h_{T} \leq c h$ in the last steps. Note that we use $W_{\overrightarrow{0}}^{1,2}(\Gamma)$ instead of $H^{1}(\Gamma)$ since the former is defined piecewise. Let $z=S\left(\bar{u}-R_{h} \bar{u}\right)$. Then we get for the second term in (7.1),

$$
\begin{aligned}
\left\|S\left(\bar{u}-R_{h} \bar{u}\right)\right\|_{L^{2}(\Omega)}^{2} & =\left(S\left(\bar{u}-R_{h} \bar{u}\right), z\right)_{L^{2}(\Omega)}=\left(\bar{u}-R_{h} \bar{u}, S^{*} z\right)_{L^{2}(\Gamma)} \\
& =\left(\bar{u}-Q_{h} \bar{u}, S^{*} z\right)_{L^{2}(\Gamma)}+\left(Q_{h} \bar{u}-R_{h} \bar{u}, S^{*} z\right)_{L^{2}(\Gamma)},
\end{aligned}
$$

where we introduced the intermediate function $Q_{h} \bar{u}$. Again, we estimate both terms in (7.3) separately. One obtains for the first term with Corollary 7.3 and $h_{T} \leq c h$,

$$
\left(\bar{u}-Q_{h} \bar{u}, S^{*} z\right)_{L^{2}(\Gamma)}=\sum_{E \in \mathcal{E}_{h}}\left(\bar{u}-Q_{h} \bar{u}, S^{*} z\right)_{L^{2}(E)} \leq c \sum_{E \in \mathcal{E}_{h}} h^{2}|\bar{u}|_{H^{1}(E)}\left|S^{*} z\right|_{H^{1}(E)}
$$

Next we apply the discrete Cauchy-Schwarz inequality, the trace theorem, the Sobolev inequality, part 2 of Lemma 2.1 and the a priori estimate from Lemma 2.3 This yields

$$
\begin{aligned}
\left(\bar{u}-Q_{h} \bar{u}, S^{*} z\right)_{L^{2}(\Gamma)} & \leq c h^{2}|\bar{u}|_{W_{\overrightarrow{0}}^{1,2}(\Gamma)}\left|S^{*} z\right|_{W_{\overrightarrow{0}}^{1,2}(\Gamma)} \leq c h^{2}|\bar{u}|_{W_{\overrightarrow{0}}^{1,2}(\Gamma)}\|P z\|_{H^{3 / 2+\epsilon}(\Omega)} \\
& \leq c h^{2}|\bar{u}|_{W_{\overrightarrow{0}}^{1,2}(\Gamma)}|| P z\left\|_{W^{2,4 / 3+\epsilon}(\Omega)} \leq c h^{2}|\bar{u}|_{W_{\overrightarrow{0}}^{1,2}(\Gamma)}\right\| P z \|_{W_{\overrightarrow{1} / 2-\bar{\epsilon}}^{2,2}(\Omega)} \\
& \leq c h^{2}|\bar{u}|_{W_{\overrightarrow{0}}^{1,2}(\Gamma)}\|z\|_{W_{\overrightarrow{1} / 2-\bar{\epsilon}}^{0,2}(\Omega)} \leq c h^{2}|\bar{u}|_{W_{\overrightarrow{0}}^{1,2}(\Gamma)}\|z\|_{L^{2}(\Omega)},
\end{aligned}
$$

which holds for $\overrightarrow{0}<\vec{\epsilon}<\overrightarrow{1} / 2-\max (0, \overrightarrow{1}-\vec{\lambda})$. For the second term in (7.3) we get with the Hölder inequality

$$
\begin{aligned}
\left(Q_{h} \bar{u}-R_{h} \bar{u}, S^{*} z\right)_{L^{2}(\Gamma)} & \leq\left\|Q_{h} \bar{u}-R_{h} \bar{u}\right\|_{L^{1}(\Gamma)}\left\|S^{*} z\right\|_{L^{\infty}(\Gamma)} \\
& \leq c\left\|Q_{h} \bar{u}-R_{h} \bar{u}\right\|_{L^{1}(\Gamma)}\|z\|_{L^{2}(\Omega)}
\end{aligned}
$$

where we used the embedding $W_{\overrightarrow{0}}^{1,2}(\Gamma) \hookrightarrow L^{\infty}(\Gamma)$ and $\left|S^{*} z\right|_{W_{\overrightarrow{0}}^{1,2}(\Gamma)} \leq c\|z\|_{L^{2}(\Omega)}$ as in (7.4). Since $R_{h} \bar{u}$ is constant on every element $E$ we can continue with

$$
\begin{array}{r}
\left\|Q_{h} \bar{u}-R_{h} \bar{u}\right\|_{L^{1}(\Gamma)}=\left\|Q_{h}\left(\bar{u}-R_{h} \bar{u}\right)\right\|_{L^{1}(\Gamma)}=\sum_{E \in \mathcal{E}_{h}}\left|\int_{E}\left(\bar{u}-R_{h} \bar{u}\right) d s\right| \\
=\sum_{j=0}^{m} \sum_{\substack{E \in \mathcal{E}_{h, j} \\
E \subset K_{1}}}\left|\int_{E}\left(\bar{u}-R_{h} \bar{u}\right) d s\right|+\sum_{j=0}^{m} \sum_{\substack{E \in \mathcal{E}_{h, j} \\
E \subset K_{2}}}\left|\int_{E}\left(\bar{u}-R_{h} \bar{u}\right) d s\right| .
\end{array}
$$


Using Lemmas 6.1 and 6.3 and Corollaries 6.2 and 6.4 we get for $\mu_{j}>1 / 4$,

$$
\begin{aligned}
& \left\|Q_{h} \bar{u}-R_{h} \bar{u}\right\|_{L^{1}(\Gamma)} \leq c\left(\sum_{\substack{E \in \mathcal{E}_{h, 0} \\
E \subset K_{1}}} h|E||\bar{u}|_{W^{1, \infty}(E)}+\sum_{j=1}^{m} \sum_{\substack{E \in \mathcal{E}_{h, j} \\
E \subset K_{1}}} h|E||\bar{u}|_{W_{1-\mu_{j}}^{1, \infty}(E)}\right. \\
& \left.+\sum_{\substack{E \in \mathcal{E}_{h, 0} \\
E \subset K_{2}}} h^{2}|E|^{1 / 2}|\bar{u}|_{W^{2,2}(E)}+\sum_{j=1}^{m} \sum_{\substack{E \in \mathcal{E}_{h, j} \\
E \subset K_{2}}} h^{2}|E|^{1 / 2}|\bar{u}|_{W_{2\left(1-\mu_{j}\right)}^{2,2}(E)}\right) \\
& \leq \operatorname{ch}\left|K_{1}\right|\left(|\bar{u}|_{W^{1, \infty}\left(K_{1} \cap \Gamma^{0}\right)}+\sum_{j=1}^{m}|\bar{u}|_{W_{1-\mu_{j}}^{1, \infty}\left(K_{1} \cap \Gamma_{j}^{ \pm}\right)}\right) \\
& +c h^{2}\left|K_{2}\right|^{1 / 2}\left(|\bar{u}|_{W^{2,2}\left(K_{2} \cap \Gamma^{0}\right)}+\sum_{j=1}^{m}|\bar{u}|_{W_{2\left(1-\mu_{j}\right)}^{2,2}\left(K_{2} \cap \Gamma_{j}^{ \pm}\right)}\right) \\
& \leq \operatorname{ch}^{2}\left(|\bar{u}|_{W_{\overrightarrow{1}-\vec{\mu}}^{1, \infty}\left(K_{1}\right)}+|\bar{u}|_{W_{2(\overrightarrow{1}-\vec{\mu})}^{2,2}\left(K_{2}\right)}\right),
\end{aligned}
$$

where we used the discrete Cauchy-Schwarz inequality and Assumption 7.1. Collecting the results from the inequalities (7.1), (17.2), (77.3), (7.4), (17.5) and (7.6) yields

$$
\left\|S_{h}\left(\bar{u}-R_{h} \bar{u}\right)\right\|_{L^{2}(\Omega)} \leq \operatorname{ch}^{2}\left(|\bar{u}|_{W_{\overrightarrow{0}}^{1,2}(\Gamma)}+|\bar{u}|_{W_{\overrightarrow{1}-\vec{\mu}}^{1, \infty}\left(K_{1}\right)}+|\bar{u}|_{W_{2(\overline{1}-\vec{\mu})}^{2,2}\left(K_{2}\right)}\right) .
$$

Next, we take into account that $\bar{u}$ is given by the projection formula (4.3). We divide the boundary $\Gamma$ into the boundary parts $\mathcal{I}$, where $\bar{u}=-\bar{p} / \nu$, and $\mathcal{A}$, where $\bar{u}=a$ or $\bar{u}=b$. We obtain for $\vec{\mu}>\overrightarrow{1} / 2$,

$$
|\bar{u}|_{W_{\overrightarrow{0}}^{1,2}(\Gamma)} \leq|\bar{u}|_{W_{\overrightarrow{0}}^{1,2}(\mathcal{I})}+|\bar{u}|_{W_{\overrightarrow{0}}^{1,2}(\mathcal{A})} \leq c|\bar{p}|_{W_{\overrightarrow{0}}^{1,2}(\mathcal{I})} \leq c\|\bar{p}\|_{W_{2(\overrightarrow{1}-\vec{\mu})}^{2,2}(\Gamma)} .
$$

The last step holds due to the embedding $W_{2(\overrightarrow{1}-\vec{\mu})}^{2,2}(\Gamma) \hookrightarrow W_{\overrightarrow{1}}^{2,2}(\Gamma) \hookrightarrow W_{\overrightarrow{0}}^{1,2}(\Gamma)$, which is valid for $\vec{\mu}>\overrightarrow{1} / 2$ (cf. parts 2 and 1 of Lemma 2.1). Analogously we get

$$
\begin{aligned}
|\bar{u}|_{W_{\overrightarrow{1}-\vec{\mu}}^{1, \infty}\left(K_{1}\right)}+|\bar{u}|_{W_{2(\overrightarrow{1}-\vec{\mu})}^{2,2}\left(K_{2}\right)} & \leq c\left(|\bar{p}|_{W_{\overrightarrow{1}-\vec{\mu}}^{1, \infty}\left(K_{1} \cap \mathcal{I}\right)}+|\bar{p}|_{W_{2(\overrightarrow{1}-\vec{\mu})}^{2,2}\left(K_{2} \cap \mathcal{I}\right)}\right) \\
& \leq c\left(|\bar{p}|_{W_{\overrightarrow{1}-\vec{\mu}}^{1, \infty}(\Gamma)}+|\bar{p}|_{W_{2(\overline{1}-\vec{\mu})}^{2,2}(\Gamma)}\right) .
\end{aligned}
$$

In summary one obtains from the inequalities (7.7), (77.8) and (7.9),

$$
\left\|S_{h}\left(\bar{u}-R_{h} \bar{u}\right)\right\|_{L^{2}(\Omega)} \leq \operatorname{ch}^{2}\left(|\bar{p}|_{W_{\overrightarrow{1}-\vec{\mu}}^{1, \infty}(\Gamma)}+\|\bar{p}\|_{W_{2(\overline{1}-\vec{\mu})}^{2,2}(\Gamma)}\right) .
$$

The results of Corollary 4.2 imply for $\overrightarrow{1} / 2<\vec{\mu}<\overrightarrow{1} / 4+\vec{\lambda} / 2$,

$$
\left\|S_{h}\left(\bar{u}-R_{h} \bar{u}\right)\right\|_{L^{2}(\Omega)} \leq \operatorname{ch}^{2}\left(\|\bar{u}\|_{L^{2}(\Gamma)}+\left\|y_{d}\right\|_{C^{0, \sigma}(\bar{\Omega})}\right) .
$$


Lemma 7.5. Let $v \in L^{2}(\Gamma)$ and $z \in L^{2}(\Omega)$. The discrete solution operators $S_{h}$ and $S_{h}^{*}$ admit for $\overrightarrow{0}<\vec{\mu} \leq \overrightarrow{1}$ the estimates

$$
\begin{aligned}
\left\|S_{h} v\right\|_{L^{2}(\Omega)} & \leq c\|v\|_{L^{2}(\Gamma)}, \\
\left\|P_{h} z\right\|_{L^{2}(\Omega)} & \leq c\|z\|_{L^{2}(\Omega)}, \\
\left\|S_{h}^{*} z\right\|_{L^{2}(\Gamma)} & \leq c\|z\|_{L^{2}(\Omega)} .
\end{aligned}
$$

Proof. We prove the second and third inequality. The first one can be proven analogously. The coercivity of the bilinear form, the Cauchy-Schwarz inequality and the embedding $H^{1}(\Omega) \hookrightarrow L^{2}(\Omega)$ yield

$$
\left\|P_{h} z\right\|_{H^{1}(\Omega)}^{2} \leq c a\left(P_{h} z, P_{h} z\right)=c\left(z, P_{h} z\right)_{L^{2}(\Omega)} \leq c\|z\|_{L^{2}(\Omega)}\left\|P_{h} z\right\|_{H^{1}(\Omega)} .
$$

One gets the second inequality with the embedding $H^{1}(\Omega) \hookrightarrow L^{2}(\Omega)$ and the third one with the trace theorem $\left\|S_{h}^{*} z\right\|_{L^{2}(\Gamma)} \leq c\left\|P_{h} z\right\|_{H^{1}(\Omega)}$.

Lemma 7.6 (Supercloseness). Let Assumption 7.1 and the condition $\overrightarrow{1} / 2<\vec{\mu}<$ $\overrightarrow{1} / 4+\vec{\lambda} / 2$ be fulfilled. Then the estimate

$$
\left\|R_{h} \bar{u}-\bar{u}_{h}\right\|_{L^{2}(\Gamma)} \leq c h^{2}|\ln h|^{3 / 2}\left(\|\bar{u}\|_{L^{2}(\Gamma)}+\left\|y_{d}\right\|_{C^{0, \sigma}(\bar{\Omega})}\right)
$$

holds true.

Proof. To prove this lemma we proceed similarly to the proof of Lemma 5.2 of [1]. In Lemma 5.1 of [1] the validity of the inequality

$$
\nu\left\|R_{h} \bar{u}-\bar{u}_{h}\right\|_{L^{2}(\Gamma)}^{2} \leq\left(R_{h} \bar{p}-\bar{p}_{h}, \bar{u}_{h}-R_{h} \bar{u}\right)_{L^{2}(\Gamma)}
$$

is stated. Inserting appropriate intermediate functions yields

$$
\begin{aligned}
\nu\left\|R_{h} \bar{u}-\bar{u}_{h}\right\|_{L^{2}(\Gamma)}^{2} & \leq\left(R_{h} \bar{p}-\bar{p}, \bar{u}_{h}-R_{h} \bar{u}\right)_{L^{2}(\Gamma)} \\
& +\left(\bar{p}-S_{h}^{*}\left(S_{h} R_{h} \bar{u}-y_{d}\right), \bar{u}_{h}-R_{h} \bar{u}\right)_{L^{2}(\Gamma)} \\
& +\left(S_{h}^{*}\left(S_{h} R_{h} \bar{u}-y_{d}\right)-\bar{p}_{h}, \bar{u}_{h}-R_{h} \bar{u}\right)_{L^{2}(\Gamma)}
\end{aligned}
$$

We are going to estimate each term on the right-hand side of (7.10) separately. Since $\bar{u}_{h}-R_{h} \bar{u}$ is constant on every boundary element $E$ we obtain for the first term,

$$
\begin{aligned}
\left(R_{h} \bar{p}-\bar{p}, \bar{u}_{h}-R_{h} \bar{u}\right)_{L^{2}(\Gamma)} & =\sum_{E \in \mathcal{E}_{h}} \int_{E}\left(R_{h} \bar{p}-\bar{p}\right)\left(\bar{u}_{h}-R_{h} \bar{u}\right) d s \\
& =\sum_{j=0}^{m} \sum_{E \in \mathcal{E}_{h, j}}\left(\bar{u}_{h}-R_{h} \bar{u}\right)_{\left.\right|_{E}} \int_{E}\left(R_{h} \bar{p}-\bar{p}\right) d s .
\end{aligned}
$$


Using Lemma 6.1 and Corollary 6.2 we can conclude as in the proof of Lemma 7.4 for $\overrightarrow{1} / 4<\vec{\mu}<\overrightarrow{1} / 4+\vec{\lambda} / 2$,

$$
\begin{aligned}
& \left(R_{h} \bar{p}-\bar{p}, \bar{u}_{h}-R_{h} \bar{u}\right)_{L^{2}(\Gamma)} \\
& \leq \operatorname{ch}^{2}\left(\sum_{E \in \mathcal{E}_{h, 0}}|E|^{1 / 2}\left|\left(\bar{u}_{h}-R_{h} \bar{u}\right)_{\left.\right|_{E}}\right||\bar{p}|_{W^{2,2}(E)}\right. \\
& \left.+\sum_{j=1}^{m} \sum_{E \in \mathcal{E}_{h, j}}|E|^{1 / 2}\left|\left(\bar{u}_{h}-R_{h} \bar{u}\right)_{\left.\right|_{E}}\right||\bar{p}|_{W_{2\left(1-\mu_{j}\right)}^{2,2}(E)}\right) \\
& =\operatorname{ch}^{2}\left(\sum_{E \in \mathcal{E}_{h, 0}}\left\|\bar{u}_{h}-R_{h} \bar{u}\right\|_{L^{2}(E)}|\bar{p}|_{W^{2,2}(E)}\right. \\
& \left.+\sum_{j=1}^{m} \sum_{E \in \mathcal{E}_{h, j}}\left\|\bar{u}_{h}-R_{h} \bar{u}\right\|_{L^{2}(E)}|\bar{p}|_{W_{2\left(1-\mu_{j}\right)}^{2,2}(E)}\right) \\
& \leq c h^{2}\left\|\bar{u}_{h}-R_{h} \bar{u}\right\|_{L^{2}(\Gamma)}|\bar{p}|_{W_{2(\overrightarrow{1}-\vec{\mu})}^{2,2}(\Gamma)} \\
& \leq \operatorname{ch}^{2}\left\|\bar{u}_{h}-R_{h} \bar{u}\right\|_{L^{2}(\Gamma)}\left(\|\bar{u}\|_{L^{2}(\Gamma)}+\left\|y_{d}\right\|_{C^{0, \sigma}(\bar{\Omega})}\right) .
\end{aligned}
$$

The last two inequalities hold with respect to the discrete Cauchy-Schwarz inequality and Corollary 4.2 For the second term in (7.10) we get with the Cauchy-Schwarz inequality

$\left(\bar{p}-S_{h}^{*}\left(S_{h} R_{h} \bar{u}-y_{d}\right), \bar{u}_{h}-R_{h} \bar{u}\right)_{L^{2}(\Gamma)} \leq\left\|\bar{p}-S_{h}^{*}\left(S_{h} R_{h} \bar{u}-y_{d}\right)\right\|_{L^{2}(\Gamma)}\left\|\bar{u}_{h}-R_{h} \bar{u}\right\|_{L^{2}(\Gamma)}$.

We again introduce intermediate functions, apply the triangle inequality and Lemma 7.5. By this we get

$$
\begin{aligned}
\| \bar{p} & -S_{h}^{*}\left(S_{h} R_{h} \bar{u}-y_{d}\right)\left\|_{L^{2}(\Gamma)}=\right\| S^{*}\left(\bar{y}-y_{d}\right)-S_{h}^{*}\left(S_{h} R_{h} \bar{u}-y_{d}\right) \|_{L^{2}(\Gamma)} \\
& \leq\left\|\left(S^{*}-S_{h}^{*}\right)\left(\bar{y}-y_{d}\right)\right\|_{L^{2}(\Gamma)}+\left\|S_{h}^{*}\left(S-S_{h}\right) \bar{u}\right\|_{L^{2}(\Gamma)}+\left\|S_{h}^{*} S_{h}\left(\bar{u}-R_{h} \bar{u}\right)\right\|_{L^{2}(\Gamma)} \\
& \leq\left\|\left(S^{*}-S_{h}^{*}\right)\left(\bar{y}-y_{d}\right)\right\|_{L^{2}(\Gamma)}+c\left\|\left(S-S_{h}\right) \bar{u}\right\|_{L^{2}(\Omega)}+c\left\|S_{h}\left(\bar{u}-R_{h} \bar{u}\right)\right\|_{L^{2}(\Gamma)} .
\end{aligned}
$$

These three terms have been estimated in Theorem 3.2, Theorem 3.1, and Lemma 7.4 . Thus, one obtains for $\overrightarrow{1} / 2<\vec{\mu}<\overrightarrow{1} / 4+\vec{\lambda} / 2<\vec{\lambda}$ and some $\epsilon \in(0,1 / 2] \cap(0, \sigma]$ with $\sigma \in(0,1)$

$$
\begin{aligned}
& \left(\bar{p}-S_{h}^{*}\left(S_{h} R_{h} \bar{u}-y_{d}\right), \bar{u}_{h}-R_{h} \bar{u}\right)_{L^{2}(\Gamma)} \\
& \leq c h^{2}|\ln h|^{3 / 2}\left(\left\|\bar{y}-y_{d}\right\|_{C^{0, \epsilon}(\bar{\Omega})}+\|\bar{y}\|_{W_{\overrightarrow{1}-\vec{\mu}}^{2,2}(\Omega)}\right. \\
& \left.\quad+\|\bar{u}\|_{L^{2}(\Gamma)}+\left\|y_{d}\right\|_{C^{0, \sigma}(\bar{\Omega})}\right)\left\|\bar{u}_{h}-R_{h} \bar{u}\right\|_{L^{2}(\Gamma)} \\
& \quad \leq c h^{2}|\ln h|^{3 / 2}\left(\|\bar{y}\|_{W_{\overrightarrow{1}-\vec{\mu}}^{2,2}(\Omega)}+\|\bar{u}\|_{L^{2}(\Gamma)}\right. \\
& \left.\quad+\left\|y_{d}\right\|_{C^{0, \sigma}(\bar{\Omega})}\right)\left\|\bar{u}_{h}-R_{h} \bar{u}\right\|_{L^{2}(\Gamma)} \\
& \leq c h^{2}|\ln h|^{3 / 2}\left(\|\bar{u}\|_{L^{2}(\Gamma)}+\left\|y_{d}\right\|_{C^{0, \sigma}(\bar{\Omega})}\right)\left\|\bar{u}_{h}-R_{h} \bar{u}\right\|_{L^{2}(\Gamma)},
\end{aligned}
$$


where we used the embedding $W_{\overrightarrow{1}-\vec{\mu}}^{2,2}(\Omega) \hookrightarrow W_{\overrightarrow{1} / 2}^{2,2}(\Omega) \hookrightarrow W^{2,2 /(2-\epsilon)}(\Omega) \hookrightarrow C^{0, \epsilon}(\bar{\Omega})$ (according to part 2 of Lemma 2.1 and the Sobolev inequality) and Theorem 4.1 Having in mind the definition of $\bar{p}_{h}$ and $S_{h}^{*}$ we get for the third term in (7.10)

$$
\begin{gathered}
\left(S_{h}^{*}\left(S_{h} R_{h} \bar{u}-y_{d}\right)-\bar{p}_{h}, \bar{u}_{h}-R_{h} \bar{u}\right)_{L^{2}(\Gamma)}=\left(S_{h}^{*}\left(S_{h}\left(R_{h} \bar{u}-\bar{u}_{h}\right)\right), \bar{u}_{h}-R_{h} \bar{u}\right)_{L^{2}(\Gamma)} \\
(7.13)=\left(S_{h}\left(R_{h} \bar{u}-\bar{u}_{h}\right), S_{h}\left(\bar{u}_{h}-R_{h} \bar{u}\right)\right)_{L^{2}(\Omega)}=-\left\|S_{h}\left(R_{h} \bar{u}-\bar{u}_{h}\right)\right\|_{L^{2}(\Omega)}^{2} \leq 0 .
\end{gathered}
$$

Finally, the inequalities (7.10), (7.11), (7.12) and (17.13) imply the desired result.

We are now prepared to formulate our main result. Let $\bar{u}$ be the solution of the optimal control problem (1.2), $\bar{y}=S \bar{u}$ the corresponding state, and $\bar{p}=P\left(S \bar{u}-y_{d}\right)$ the corresponding adjoint state; see Theorem 4.1 Furthermore, let $\bar{u}_{h}, \bar{y}_{h}$ and $\bar{p}_{h}$ be their discrete counterparts as introduced in Section 5] see Lemma 5.1. We define the projection $\tilde{u}_{h}$ of $\bar{p}_{h}$ by

$$
\tilde{u}_{h}=\Pi_{[a, b]}\left(-\frac{1}{\nu} \bar{p}_{h}\right) .
$$

This function $\tilde{u}_{h}$ is piecewise linear and continuous, but it does not belong to the finite element space $\left.V_{h}\right|_{\Gamma}$ in general. However, one can prove that $\tilde{u}_{h}$ possesses superconvergence properties.

Theorem 7.7. Under Assumption 7.1 the discretization error estimates

$$
\begin{aligned}
&\left\|\bar{y}-\bar{y}_{h}\right\|_{L^{2}(\Omega)} \leq c h^{2}|\ln h|^{3 / 2}\left(\|\bar{u}\|_{L^{2}(\Gamma)}+\left\|y_{d}\right\|_{C^{0, \sigma}(\bar{\Omega})}\right), \\
&\left\|\bar{p}-\bar{p}_{h}\right\|_{L^{2}(\Gamma)}+\left\|\bar{p}-\bar{p}_{h}\right\|_{L^{2}(\Omega)} \leq c h^{2}|\ln h|^{3 / 2}\left(\|\bar{u}\|_{L^{2}(\Gamma)}+\left\|y_{d}\right\|_{C^{0, \sigma}(\bar{\Omega})}\right), \\
&\left\|\bar{u}-\tilde{u}_{h}\right\|_{L^{2}(\Gamma)} \leq c h^{2}|\ln h|^{3 / 2}\left(\|\bar{u}\|_{L^{2}(\Gamma)}+\left\|y_{d}\right\|_{C^{0, \sigma}(\bar{\Omega})}\right)
\end{aligned}
$$

hold, provided that the grading parameter $\vec{\mu}$ fulfills the condition $\overrightarrow{1} / 2<\vec{\mu}<$ $\overrightarrow{1} / 4+\vec{\lambda} / 2$.

Proof. Let $\epsilon \in(0,1 / 2] \cap(0, \sigma]$ with $\sigma \in(0,1)$ and $\overrightarrow{1} / 2<\vec{\mu}<\overrightarrow{1} / 4+\vec{\lambda} / 2$. Introducing intermediate functions, the triangle inequality and Lemma 7.5 yield

$$
\begin{aligned}
\left\|\bar{y}-\bar{y}_{h}\right\|_{L^{2}(\Omega)} & \leq\left\|\left(S-S_{h}\right) \bar{u}\right\|_{L^{2}(\Omega)}+\left\|S_{h}\left(\bar{u}-R_{h} \bar{u}\right)\right\|_{L^{2}(\Omega)}+\left\|S_{h}\left(R_{h} \bar{u}-\bar{u}_{h}\right)\right\|_{L^{2}(\Omega)} \\
& \leq\left\|\left(S-S_{h}\right) \bar{u}\right\|_{L^{2}(\Omega)}+\left\|S_{h}\left(\bar{u}-R_{h} \bar{u}\right)\right\|_{L^{2}(\Omega)}+c\left\|R_{h} \bar{u}-\bar{u}_{h}\right\|_{L^{2}(\Gamma)} .
\end{aligned}
$$

If we apply the results of Theorem 3.1] Lemma 7.4 Lemma 7.6 and Theorem 4.1 we obtain

$$
\begin{aligned}
\left\|\bar{y}-\bar{y}_{h}\right\|_{L^{2}(\Omega)} & \leq c h^{2}|\ln h|^{3 / 2}\left(\|\bar{y}\|_{W_{\overline{1}-\vec{\mu}}^{2,2}(\Omega)}+\|\bar{u}\|_{L^{2}(\Gamma)}+\left\|y_{d}\right\|_{C^{0, \sigma}(\bar{\Omega})}\right) \\
& \leq c h^{2}|\ln h|^{3 / 2}\left(\|\bar{u}\|_{L^{2}(\Gamma)}+\left\|y_{d}\right\|_{C^{0, \sigma}(\bar{\Omega})}\right) .
\end{aligned}
$$

The error of the adjoint state on the boundary and in the domain can be estimated by

$$
\begin{aligned}
& \left\|\bar{p}-\bar{p}_{h}\right\|_{L^{2}(\Gamma)}+\left\|\bar{p}-\bar{p}_{h}\right\|_{L^{2}(\Omega)} \leq\left\|\left(S^{*}-S_{h}^{*}\right)\left(\bar{y}-y_{d}\right)\right\|_{L^{2}(\Gamma)}+\left\|S_{h}^{*}\left(\bar{y}-\bar{y}_{h}\right)\right\|_{L^{2}(\Gamma)} \\
& \quad+\left\|\left(P-P_{h}\right)\left(\bar{y}-y_{d}\right)\right\|_{L^{2}(\Omega)}+\left\|P_{h}\left(\bar{y}-y_{h}\right)\right\|_{L^{2}(\Omega)}, \\
& \quad \leq\left\|\left(S^{*}-S_{h}^{*}\right)\left(\bar{y}-y_{d}\right)\right\|_{L^{2}(\Gamma)}+\left\|\left(P-P_{h}\right)\left(\bar{y}-y_{d}\right)\right\|_{L^{2}(\Omega)} \\
& \quad+\left\|\bar{y}-\bar{y}_{h}\right\|_{L^{2}(\Omega)},
\end{aligned}
$$


where we used Lemma [7.5. Next, we apply Theorem 3.1] Theorem 3.2 and (7.14). By this we get

$$
\begin{aligned}
& \left\|\bar{p}-\bar{p}_{h}\right\|_{L^{2}(\Gamma)}+\left\|\bar{p}-\bar{p}_{h}\right\|_{L^{2}(\Omega)} \\
& \quad \leq c h^{2}|\ln h|^{3 / 2}\left(\left\|\bar{y}-y_{d}\right\|_{C^{0, \epsilon}(\bar{\Omega})}+\left\|\bar{y}-y_{d}\right\|_{W_{\overline{1}-\vec{\mu}}^{0,2}(\Omega)}+\|\bar{u}\|_{L^{2}(\Gamma)}+\left\|y_{d}\right\|_{C^{0, \sigma}(\bar{\Omega})}\right) \\
& \quad \leq c h^{2}|\ln h|^{3 / 2}\left(\|\bar{y}\|_{C^{0, \epsilon}(\bar{\Omega})}+\|\bar{y}\|_{W_{\overrightarrow{1}-\vec{\mu}}^{0,2}(\Omega)}+\|\bar{u}\|_{L^{2}(\Gamma)}+\left\|y_{d}\right\|_{W_{\overrightarrow{1}-\vec{\mu}}^{0,2}(\Omega)}+\left\|y_{d}\right\|_{C^{0, \sigma}(\bar{\Omega})}\right) .
\end{aligned}
$$

The embeddings $W_{\overrightarrow{1}-\vec{\mu}}^{2,2}(\Omega) \hookrightarrow W_{\overrightarrow{1} / 2}^{2,2}(\Omega) \hookrightarrow W^{2,2 /(2-\epsilon)}(\Omega) \hookrightarrow C^{0, \epsilon}(\bar{\Omega}) \hookrightarrow W_{\overrightarrow{1}-\vec{\mu}}^{0,2}(\Omega)$, which hold according to part 2 of Lemma 2.1 and the Sobolev embedding theorem, yield together with Theorem 4.1

$$
\left\|\bar{p}-\bar{p}_{h}\right\|_{L^{2}(\Gamma)}+\left\|\bar{p}-\bar{p}_{h}\right\|_{L^{2}(\Omega)} \leq c h^{2}|\ln h|^{3 / 2}\left(\|\bar{u}\|_{L^{2}(\Gamma)}+\left\|y_{d}\right\|_{C^{0, \sigma}(\bar{\Omega})}\right) .
$$

Finally, we observe that the projection operator $\Pi_{[a, b]}$ is Lipschitz continuous (cf. also the proof of Theorem 4.1). This implies together with (7.15),

$$
\begin{aligned}
\nu\left\|\bar{u}-\tilde{u}_{h}\right\|_{L^{2}(\Gamma)} & =\nu\left\|\Pi_{[a, b]}\left(-\frac{1}{\nu} \bar{p}\right)-\Pi_{[a, b]}\left(-\frac{1}{\nu} \bar{p}_{h}\right)\right\|_{L^{2}(\Gamma)} \leq c\left\|\bar{p}-\bar{p}_{h}\right\|_{L^{2}(\Gamma)} \\
& \leq c h^{2}|\ln h|^{3 / 2}\left(\|\bar{u}\|_{L^{2}(\Gamma)}+\left\|y_{d}\right\|_{C^{0, \sigma}(\bar{\Omega})}\right),
\end{aligned}
$$

which ends the proof.

Remark 7.8. An alternative discretization concept for optimal control problems is variational discretization. It is introduced in [12] for distributed control problems and applied in [6] for Neumann boundary control problems. The finite element error estimate on the boundary given in Theorem 3.2 can be used to prove the same discretization error estimates of order two (up to a logarithmic factor) for this concept, compare Section 7 in [20] and Section 6 in [1].

Remark 7.9. In our former paper [1] we proved error estimates of order $\frac{3}{2}$ for $\left\|\bar{y}-\bar{y}_{h}\right\|_{L^{2}(\Omega)},\left\|\bar{p}-\bar{p}_{h}\right\|_{L^{2}(\Gamma)},\left\|\bar{p}-\bar{p}_{h}\right\|_{L^{2}(\Omega)}$, and $\left\|\bar{u}-\tilde{u}_{h}\right\|_{L^{2}(\Gamma)}$ by using simpler techniques and a weaker grading condition. The numerical tests in Section 7 of 1 ] showed that a stronger mesh grading leads to almost second order convergence; see [1. Table 1], and initiated the research of the current paper. Hence the numerical example there can be considered as an example for the theory developed here.

\section{Proof of Regularity Results}

The aim of this section is to prove Lemma 2.3 and Lemma 2.4 To this end, it is useful to have a second type of weighted Sobolev space and appropriate weighted Hölder spaces available.

The spaces $V_{\vec{\beta}}^{k, p}(\Omega)$ and $V_{\vec{\beta}}^{k, p}(\Gamma)$ are defined analogously to the weighted Sobolev spaces $W_{\vec{\beta}}^{k, p}(\Omega)$ and $W_{\vec{\beta}}^{k, p}(\Gamma)$, respectively (see Section 2), except that the weighting functions $r_{j}^{\beta_{j}}$ in the definition of the norms are substituted by $r_{j}^{\beta_{j}-k+|\alpha|}$. Note that the the classical Sobolev spaces $W^{k, p}(\Omega)$ are included in the weighted Sobolev spaces $W_{\vec{\beta}}^{k, p}(\Omega)$ by setting $\beta_{j}=0$ for $j=1, \ldots, m$, whereas they do not belong to the scale of the weighted spaces $V_{\vec{\beta}}^{k, p}(\Omega)$. But there is a relation between the space 
$W_{\vec{\beta}}^{k, p}(\Omega)$ and the space $V_{\vec{\beta}}^{k, p}(\Omega)$. We recall Lemma 2.1 of [1], which represents this relation for $k=p=2$. For general $k \in \mathbb{N}_{0}$ and $1<p<\infty$ we refer to Theorem 2.1 of $[22$.

Lemma 8.1. Let $\eta_{j}, j=1, \ldots, m$, be infinitely differentiable cut-off functions in $\bar{\Omega}$ equal to one in $\Omega_{R_{j} / 64}$ and $\operatorname{supp} \eta_{j} \subset \Omega_{R_{j}}$. For $\vec{\beta} \in(0,1)^{m}$ one has

$$
W_{\vec{\beta}}^{2,2}(\Omega)=V_{\vec{\beta}}^{2,2}(\Omega) \oplus \eta_{1} \mathcal{P}_{0}(\Omega) \oplus \cdots \oplus \eta_{m} \mathcal{P}_{0}(\Omega),
$$

where $\mathcal{P}_{0}(\Omega)$ is the set of constant polynomials on $\Omega$. In particular, for any $v \in W_{\vec{\beta}}^{2,2}(\Omega)$ one can write $v=v_{s}+\sum_{j=1}^{m} \eta_{j} v\left(x^{(j)}\right)$ with $v_{s} \in V_{\vec{\beta}}^{2,2}(\Omega)$. Moreover, the norm equivalence

$$
\|v\|_{W_{\vec{\beta}}^{2,2}(\Omega)} \sim\left\|v_{s}\right\|_{V_{\vec{\beta}}^{2,2}(\Omega)}+\sum_{j=1}^{m}\left|v\left(x^{(j)}\right)\right|
$$

is valid.

Finally, we introduce the weighted Hölder spaces $N_{\vec{\beta}}^{k, \sigma}(\Omega)$, where $k \in \mathbb{N}_{0}, \sigma \in$ $(0,1)$ and $\vec{\beta}=\left(\beta_{1}, \ldots, \beta_{m}\right)^{T} \in \mathbb{R}^{m}$. These spaces consist of all $k$ times continuously differentiable functions in $\bar{\Omega} \backslash \mathcal{C}$ such that

$$
\|v\|_{N_{\vec{\beta}}^{k, \sigma}(\Omega)} \sim\|v\|_{C^{k, \sigma}\left(\bar{\Omega}^{0}\right)}+\sum_{j=1}^{m}\|v\|_{N_{\beta_{j}}^{k, \sigma}\left(\Omega_{R_{j}}\right)}
$$

is finite, where

$$
\begin{aligned}
\|v\|_{N_{\beta_{j}}^{k, \sigma}\left(\Omega_{R_{j}}\right)} & =\sum_{|\alpha| \leq k}\left\|r_{j}^{\beta_{j}-\sigma-k+|\alpha|} D^{\alpha} v\right\|_{C^{0}\left(\bar{\Omega}_{R_{j}}\right)} \\
& +\sum_{|\alpha|=k} \sup _{x, y \in \Omega_{R_{j}}} \frac{\left|r_{j}(x)^{\beta_{j}} D_{x}^{\alpha} v(x)-r_{j}(y)^{\beta_{j}} D_{y}^{\alpha} v(y)\right|}{|x-y|^{\sigma}} .
\end{aligned}
$$

The trace spaces $N_{\vec{\beta}}^{k, \sigma}(\Gamma)$ are defined in the same manner.

Now we have everything at hand to discuss the regularity results. If the boundary of the domain $\Omega$ is smooth enough, and if $f \in L^{2}(\Omega)$ and $g \in H^{1 / 2}(\Gamma)$, then one can show that the generalized solution is an element of $H^{2}(\Omega)$. In polygonal domains this statement fails. In general the weak solution of (1.1) does not belong to $H^{2}(\Omega)$ if $\omega_{j}>\pi$ for some $j$. Instead, one can show that the solution has the asymptotics

$$
y=\sum_{j=1}^{m} \eta_{j} c_{0, j}+\sum_{j: \omega_{j}>\pi} \eta_{j} c_{1, j} r_{j}^{\lambda_{j}} \cos \left(\lambda_{j} \varphi_{j}\right)+y_{\mathrm{reg}},
$$

where $\eta_{j}$ denote the cut-off functions introduced in Lemma 8.1 $c_{0, j}$ and $c_{1, j}$ are some constants, $\lambda_{j}=\pi / \omega_{j}$ and the function $y_{\text {reg }}$ belongs to $H^{2}(\Omega)$. For more general $f \in W_{\vec{\beta}}^{0,2}(\Omega)$ and $g \in W_{\vec{\beta}}^{1 / 2,2}(\Gamma)$ with $\max \left(0,1-\lambda_{j}\right)<\beta_{j}<1$ the asymptotic representation

$$
y=\sum_{j=1}^{m} \eta_{j} c_{0, j}+y_{\text {sing }}
$$


holds, where $y_{\text {sing }}$ belongs to $V_{\vec{\beta}}^{2,2}(\Omega)$ (cf. [25, Chapter 2]). Based on such representations, regularity results in weighted Sobolev spaces can be proven. The result needed is stated in Lemma 2.3. We will now sketch the proof.

Proof. (Lemma 2.3) We get from the Lax-Milgram Theorem the unique solvability of problem (1.1) in $H^{1}(\Omega)$ if $\beta_{j}<1$. The unique solvability in $W_{\vec{\beta}}^{2,2}(\Omega)$ and the validity of the a priori estimate for $\max \left(0,1-\lambda_{j}\right)<\beta_{j}<1$ is then a consequence of Lemma 6.3 .3 of [21] by using a partition of unity method (the aforementioned asymptotic representation is used in the proof of that lemma). In the case that $\beta_{j}=0$ and $1-\lambda_{j}<0$ we can deduce the unique solvability from Corollary 4.4.3.8 of [11. The a priori estimate holds in that case according to Theorem 4.3.1.4 of [11] and the Lax-Milgram Theorem.

Next, we would like to prove also regularity results in $W_{\vec{\beta}}^{2, \infty}(\Omega)$ and $W_{\vec{\beta}}^{2, \infty}(\Gamma)$, as stated in Lemma 2.4. To our knowledge there is no reference where this is done directly. Instead, we use regularity results in weighted Hölder spaces for that purpose. The following lemma represents parts of Theorem 1.4.5 of [17], which has been adapted to our setting (compare also [22]). Note, that asymptotic representations of the solution are again used in its proof.

Lemma 8.2. Let $u \in V_{\vec{\beta}}^{2,2}(\Omega)$ with $\beta_{j}=1 / 2$ for $j=1, \ldots, m$ be a solution of

$$
\begin{aligned}
-\Delta u+u=F & \text { in } \Omega, \\
\partial_{n} u=G & \text { on } \Gamma_{j}, \quad j=1, \ldots, m,
\end{aligned}
$$

where $F \in N_{\vec{\delta}}^{0, \sigma}(\Omega)$ and $G \in N_{\vec{\delta}}^{1, \sigma}(\Gamma)$. If $0<2+\sigma-\delta_{j}<\lambda_{j}$ for $j=1, \ldots, m$, then $u$ belongs to $N_{\vec{\delta}}^{2, \sigma}(\Omega)$ and the a priori estimate

$$
\|u\|_{N_{\vec{\delta}}^{2, \sigma}(\Omega)} \leq c\left(\|F\|_{N_{\vec{\delta}}^{0, \sigma}(\Omega)}+\|G\|_{N_{\vec{\delta}}^{1, \sigma}(\Gamma)}+\|u\|_{L^{1}(\Omega)}\right)
$$

is valid.

We are now prepared to prove Lemma 2.4

Proof (Lemma 2.4). From Lemma 2.3 we know that the solution $y$ of (1.1) belongs to $W_{\vec{\beta}}^{2,2}(\Omega)$ if $1>\beta_{j}>\max \left(0,1-\lambda_{j}\right)$. In the following we choose $\beta_{j}=\frac{1}{2}$, which is possible since $\lambda_{j}>1 / 2$ for every $\omega_{j} \in[0,2 \pi)$. Next, we would like to apply Lemma 8.2 but $y \notin V_{\vec{\beta}}^{2,2}(\Omega)$. Instead, we first use Lemma 8.1 This yields the splitting

$$
u:=y-\sum_{j=1}^{m} \eta_{j} y\left(x^{(j)}\right) \in V_{\vec{\beta}}^{2,2}(\Omega),
$$

where $\eta_{j}$ denote the cut-off functions introduced in Lemma 8.1 Furthermore, $u$ solves

$$
\begin{aligned}
-\Delta u+u & =f-\sum_{j=1}^{m} \eta_{j} y\left(x^{(j)}\right)+\sum_{j=1}^{m} y\left(x^{(j)}\right) \Delta \eta_{j}=: F & & \text { in } \Omega, \\
\partial_{n} u & =-\sum_{j=1}^{m} y\left(x^{(j)}\right) \partial_{n} \eta_{j}=: G & & \text { on } \Gamma_{k}, k=1, \ldots, m .
\end{aligned}
$$


Let $\delta_{j} \geq \sigma$ be real numbers for $j=1, \ldots, m$. Then, one can show for any function $w \in C^{0, \sigma}(\bar{\Omega})$ that

$$
\begin{aligned}
\|w\|_{N_{\bar{\delta}}^{0, \sigma}(\Omega)} \leq & \|w\|_{C^{0, \sigma}\left(\bar{\Omega}_{0}\right)} \\
& +c \sum_{j=1}^{m}\left(\left\|r_{j}^{\delta_{j}-\sigma} w\right\|_{C^{0}\left(\bar{\Omega}_{R_{j}}\right)}+\sup _{\substack{x, y \in \Omega_{R_{j}} \\
|x-y| \leq \frac{1}{2} r_{j}(x)}} r_{j}(x)^{\delta_{j}} \frac{|w(x)-w(y)|}{|x-y|^{\sigma}}\right) \\
\leq & c\|w\|_{C^{0, \sigma}(\bar{\Omega})}
\end{aligned}
$$

(cf. Section 1.1 in [27] for technical details or Section 5 of [22]). Thus, the functions $f, \eta_{j}$ and $\Delta \eta_{j}$ belong to $N_{\vec{\delta}}^{0, \sigma}(\Omega)$ and $\partial_{n} \eta_{j}$ to $N_{\vec{\delta}}^{1, \sigma}(\Gamma)\left(\Delta \eta_{j}\right.$ and $\partial_{n} \eta_{j}$ even vanish in the neighborhood of every corner). Based on this we can conclude for $\delta_{j}-\sigma \geq 0$,

$$
F \in N_{\vec{\delta}}^{0, \sigma}(\Omega) \text { and } G \in N_{\vec{\delta}}^{1, \sigma}(\Gamma) .
$$

Now we can apply Lemma 8.2 We obtain that $u$ belongs to $N_{\vec{\delta}}^{2, \sigma}(\Omega)$ if $0<2+\sigma-$ $\delta_{j}<\lambda_{j}$ for $j=1, \ldots, m$. Furthermore, the a priori estimate

$$
\|u\|_{N_{\vec{\delta}}^{2, \sigma}(\Omega)} \leq c\left(\|F\|_{N_{\vec{\delta}}^{0, \sigma}(\Omega)}+\|G\|_{N_{\vec{\delta}}^{1, \sigma}(\Gamma)}+\|u\|_{L^{1}(\Omega)}\right)
$$

is valid. By setting $\gamma_{j}=\delta_{j}-\sigma \geq 0$ we obtain

$$
\begin{aligned}
\|y\|_{W_{\vec{\gamma}}^{2, \infty}(\Omega)} & +\|y\|_{W_{\vec{\gamma}}^{2, \infty}(\Gamma)} \leq c\left(\|y\|_{C^{2}\left(\bar{\Omega}^{0}\right)}+\sum_{j=1}^{m} \sum_{|\alpha| \leq 2}\left\|r_{j}^{\gamma_{j}} D^{\alpha} y\right\|_{C^{0}\left(\bar{\Omega}_{R_{j}}\right)}\right) \\
& \leq c\left(\|u\|_{C^{2}\left(\bar{\Omega}^{0}\right)}+\sum_{j=1}^{m} \sum_{|\alpha| \leq 2}\left\|r_{j}^{\gamma_{j}} D^{\alpha} u\right\|_{C^{0}\left(\bar{\Omega}_{R_{j}}\right)}\right. \\
& \left.+\sum_{j=1}^{m}\left|y\left(x^{(j)}\right)\right|\left[\left\|\eta_{j}\right\|_{C^{2}\left(\bar{\Omega}^{0}\right)}+\sum_{|\alpha| \leq 2}\left\|r_{j}^{\gamma_{j}} D^{\alpha} \eta_{j}\right\|_{C^{0}\left(\bar{\Omega}_{R_{j}}\right)}\right]\right) \\
& \leq c\left(\|u\|_{C^{2}\left(\bar{\Omega}^{0}\right)}+\sum_{j=1}^{m} \sum_{|\alpha| \leq 2}\left\|r_{j}^{\gamma_{j}} D^{\alpha} u\right\|_{C^{0}\left(\bar{\Omega}_{R_{j}}\right)}+\sum_{j=1}^{m}\left|y\left(x^{(j)}\right)\right|\right)
\end{aligned}
$$

where we inserted (8.3) and used that $r_{j}^{\gamma_{j}}$ (for $\gamma_{j} \geq 0$ ) and $\left|D^{\alpha} \eta_{j}\right|$ is bounded by a constant. Since $\gamma_{j}=\delta_{j}-\sigma$ and $2-|\alpha| \geq 0$ for $|\alpha| \leq 2$ we can conclude

$$
\begin{aligned}
\|y\|_{W_{\vec{\gamma}}^{2, \infty}(\Omega)} & +\|y\|_{W_{\vec{\gamma}}^{2, \infty}(\Gamma)} \\
& \leq c\left(\|u\|_{C^{2}\left(\bar{\Omega}^{0}\right)}+\sum_{j=1}^{m} \sum_{|\alpha| \leq 2}\left\|r_{j}^{\delta_{j}-\sigma-2+|\alpha|} D^{\alpha} u\right\|_{C^{0}\left(\bar{\Omega}_{R_{j}}\right)}+\sum_{j=1}^{m}\left|y\left(x^{(j)}\right)\right|\right) \\
(8.6) & \leq c\left(\|u\|_{N_{\vec{\delta}}^{2, \sigma}(\Omega)}+\sum_{j=1}^{m}\left|y\left(x^{(j)}\right)\right|\right) .
\end{aligned}
$$


Next, we apply the a priori estimate (8.5), which holds for $\gamma_{j}>2-\lambda_{j}$, and insert the definitions of $F$ and $G$ and (8.3). This yields

$$
\begin{aligned}
\|u\|_{N_{\vec{\delta}}^{2, \sigma}(\Omega)} \leq c(\| f & +\sum_{j=1}^{m} y\left(x^{(j)}\right)\left(\Delta \eta_{j}-\eta_{j}\right)\left\|_{N_{\vec{\delta}}^{0, \sigma}(\Omega)}+\right\| \sum_{j=1}^{m} y\left(x^{(j)}\right) \partial_{n} \eta_{j} \|_{N_{\vec{\delta}}^{1, \sigma}(\Gamma)} \\
& \left.+\left\|y-\sum_{j=1}^{m} \eta_{j} y\left(x^{(j)}\right)\right\|_{L^{1}(\Omega)}\right) \\
& \leq c\left(\|f\|_{N_{\vec{\delta}}^{0, \sigma}(\Omega)}+\|y\|_{L^{1}(\Omega)}+\sum_{j=1}^{m}\left|y\left(x^{(j)}\right)\right|\right) .
\end{aligned}
$$

Again we used that $\left|D^{\alpha} \eta_{j}\right|$ is bounded by a constant. The last two terms in (8.7) can be estimated by applying the Sobolev embedding theorem, part 2 of Lemma 2.1] and the a priori estimate of Lemma 2.3. By this we obtain with some $\epsilon \in(1,4 / 3)$ (see Section 9),

$$
\begin{aligned}
\|y\|_{L^{1}(\Omega)}+\sum_{j=1}^{m}\left|y\left(x^{(j)}\right)\right| & \leq c\|y\|_{C^{0}(\bar{\Omega})} \leq c\|y\|_{W^{2,1+\epsilon}(\bar{\Omega})} \leq c\|y\|_{W_{\vec{\beta}}^{2,2}(\bar{\Omega})} \\
& \leq c\|f\|_{W_{\vec{\beta}}^{0,2}(\bar{\Omega})} \leq c\|f\|_{L^{2}(\Omega)} \leq c\|f\|_{C^{0, \sigma}(\bar{\Omega})} .
\end{aligned}
$$

Finally, the inequalities (8.4), (8.6), (8.7) and (8.8) yield the a priori estimate of the assertion.

\section{Added AFter PROOF}

(1) On page 43, line 2 below equation (3.14), the choice $q^{\prime}=4 q /(2 q+2+\alpha q)>1$ should be replaced by $q^{\prime}=2 q^{2} /(4 q-2+\alpha q(q-1))>1$, and on the next line the embedding $W_{\alpha}^{2, q}(\hat{T}) \hookrightarrow W^{2, q^{\prime}}(\hat{T})$ should be replaced by $W_{\alpha}^{0, q}(\hat{T}) \hookrightarrow$ $W^{0, q^{\prime}}(\hat{T})$.

(2) On page 56. lines 3 and 4 below equation (6.3), the quantity $4 /\left(5-4 \mu_{j}\right)$ should be replaced by $8 /\left(9-4 \mu_{j}\right)$.

(3) On page68 line 3 below equation (8.7), It should read $\epsilon \in(0,1 / 3)$, instead of $\epsilon \in(1,4 / 3)$.

\section{ACKNOWLEDGEMEnTs}

The authors would like to thank Anna-Margarete Sändig for fruitful discussions about the proof of Lemma 2.4 and an unknown referee for many hints to improve the paper. They further acknowledge the support of the DFG priority program 1253 "Optimization with partial differential equations".

\section{REFERENCES}

[1] Thomas Apel, Johannes Pfefferer, and Arnd Rösch, Finite element error estimates for Neumann boundary control problems on graded meshes, Comput. Optim. Appl. 52 (2012), no. 1, 3-28, DOI 10.1007/s10589-011-9427-x. MR2925763

[2] Thomas Apel, Arnd Rösch, and Dieter Sirch, $L^{\infty}$-error estimates on graded meshes with application to optimal control, SIAM J. Control Optim. 48 (2009), no. 3, 1771-1796, DOI 10.1137/080731724. MR2516188 (2010f:49064) 
[3] Thomas Apel, Arnd Rösch, and Gunter Winkler, Optimal control in non-convex domains: a priori discretization error estimates, Calcolo 44 (2007), no. 3, 137-158, DOI 10.1007/s10092007-0133-0. MR2352719(2008j:49083)

[4] Thomas Apel and Dieter Sirch, $L^{2}$-error estimates for Dirichlet and Neumann problems on anisotropic finite element meshes, Appl. Math. 56 (2011), no. 2, 177-206, DOI 10.1007/s10492-011-0002-7. MR2810243 (2012e:65258)

[5] Susanne C. Brenner and L. Ridgway Scott, The Mathematical Theory of Finite Element Methods, 3rd ed., Texts in Applied Mathematics, vol. 15, Springer, New York, 2008. MR2373954(2008m:65001)

[6] Eduardo Casas and Mariano Mateos, Error estimates for the numerical approximation of Neumann control problems, Comput. Optim. Appl. 39 (2008), no. 3, 265-295, DOI 10.1007/s10589-007-9056-6. MR2396868 (2009a:49055)

[7] Eduardo Casas, Mariano Mateos, and Fredi Tröltzsch, Error estimates for the numerical approximation of boundary semilinear elliptic control problems, Comput. Optim. Appl. 31 (2005), no. 2, 193-219, DOI 10.1007/s10589-005-2180-2. MR.2150243 (2006d:49077)

[8] Monique Dauge, Elliptic Boundary Value Problems on Corner Domains, Lecture Notes in Mathematics, vol. 1341, Springer-Verlag, Berlin, 1988. Smoothness and asymptotics of solutions. MR961439 (91a:35078)

[9] Alan Demlow, Johnny Guzmán, and Alfred H. Schatz, Local energy estimates for the finite element method on sharply varying grids, Math. Comp. 80 (2011), no. 273, 1-9, DOI 10.1090/S0025-5718-2010-02353-1. MR2728969(2012a:65324)

[10] J. Deny and J. L. Lions, Les espaces du type de Beppo Levi (French), Ann. Inst. Fourier, Grenoble 5 (1953), 305-370 (1955). MR0074787(17,646a)

[11] P. Grisvard. Elliptic Problems in Nonsmooth Domains. Pitman, Boston, 1985.

[12] M. Hinze, A variational discretization concept in control constrained optimization: the linearquadratic case, Comput. Optim. Appl. 30 (2005), no. 1, 45-61, DOI 10.1007/s10589-005-45595. MR2122182 (2005i:49034)

[13] Michael Hinze and Ulrich Matthes, A note on variational discretization of elliptic Neumann boundary control, Control Cybernet. 38 (2009), no. 3, 577-591. MR2650352 (2011c:49059)

[14] M. Hinze, R. Pinnau, M. Ulbrich, and S. Ulbrich, Optimization with PDE constraints, Mathematical Modelling: Theory and Applications, vol. 23, Springer, New York, 2009. MR2516528 (2010h:49002)

[15] David S. Jerison and Carlos E. Kenig, The Neumann problem on Lipschitz domains, Bull. Amer. Math. Soc. (N.S.) 4 (1981), no. 2, 203-207, DOI 10.1090/S0273-0979-1981-14884-9. MR598688 (84a:35064)

[16] V. A. Kozlov, V. G. Maz'ya, and J. Rossmann, Elliptic Boundary Value Problems in Domains with Point singularities, Mathematical Surveys and Monographs, vol. 52, American Mathematical Society, Providence, RI, 1997. MR.1469972 (98f:35038)

[17] V. A. Kozlov, V. G. Maz'ya, and J. Rossmann, Spectral Problems Associated with Corner Singularities of Solutions to Elliptic Equations, Mathematical Surveys and Monographs, vol. 85, American Mathematical Society, Providence, RI, 2001. MR.1788991 (2001i:35069)

[18] Alois Kufner and Anna-Margarete Sändig, Some Applications of Weighted Sobolev Spaces, Teubner-Texte zur Mathematik [Teubner Texts in Mathematics], vol. 100, BSB B. G. Teubner Verlagsgesellschaft, Leipzig, 1987. With German, French and Russian summaries. MR.926688 (89h:35096)

[19] Hengguang Li, Anna Mazzucato, and Victor Nistor, Analysis of the finite element method for transmission/mixed boundary value problems on general polygonal domains, Electron. Trans. Numer. Anal. 37 (2010), 41-69. MR2777235 (2012c:65195)

[20] Mariano Mateos and Arnd Rösch, On saturation effects in the Neumann boundary control of elliptic optimal control problems, Comput. Optim. Appl. 49 (2011), no. 2, 359-378, DOI 10.1007/s10589-009-9299-5. MR2795722 (2012e:49067)

[21] Vladimir Maz'ya and Jürgen Rossmann, Elliptic Equations in Polyhedral Domains, Mathematical Surveys and Monographs, vol. 162, American Mathematical Society, Providence, RI, 2010. MR2641539(2011h:35002)

[22] V. G. Maz'ya and B. A. Plamenevsky, Weighted spaces with nonhomogeneous norms and boundary value problems in domains with conical points. American Mathematical Society Translations, 123(2):89-107, 1984. 
[23] J. M. Melenk and B. Wohlmuth, Quasi-optimal approximation of surface based Lagrange multipliers in finite element methods, SIAM J. Numer. Anal. 50 (2012), no. 4, 2064-2087, DOI 10.1137/110832999. MR3022210

[24] C. Meyer and A. Rösch, Superconvergence properties of optimal control problems, SIAM J. Control Optim. 43 (2004), no. 3, 970-985 (electronic), DOI 10.1137/S0363012903431608. MR2114385 (2005i:49024)

[25] Sergey A. Nazarov and Boris A. Plamenevsky, Elliptic Problems in Domains with Piecewise Smooth Boundaries, de Gruyter Expositions in Mathematics, vol. 13, Walter de Gruyter \& Co., Berlin, 1994. MR1283387 (95h:35001)

[26] J. Pfefferer and K. Krumbiegel, Superconvergence for Neumann boundary control problems governed by semilinear elliptic equations, arXiv:1311:6282 [math.NA].

[27] J. Roßmann. Gewichtete Sobolev-Slobodetskij-Räume und Anwendungen auf elliptische Randwertprobleme in Gebieten mit Kanten. Habilitationsschrift, Universität Rostock, 1988.

[28] A. H. Schatz and L. B. Wahlbin, Interior maximum norm estimates for finite element methods, Math. Comp. 31 (1977), no. 138, 414-442. MR0431753 (55 \#4748)

[29] A. H. Schatz and L. B. Wahlbin, Maximum norm estimates in the finite element method on plane polygonal domains. I, Math. Comp. 32 (1978), no. 141, 73-109. MR0502065 (58 \#19233a)

[30] A. H. Schatz and L. B. Wahlbin, Maximum norm estimates in the finite element method on plane polygonal domains. II. Refinements, Math. Comp. 33 (1979), no. 146, 465-492. MR0502067 (58 \#19233b)

[31] Fredi Tröltzsch, Optimal Control of Partial Differential Equations, Graduate Studies in Mathematics, vol. 112, American Mathematical Society, Providence, RI, 2010. Theory, methods and applications; Translated from the 2005 German original by Jürgen Sprekels. MR 2583281 (2011h:49004)

[32] Lars B. Wahlbin, Local behavior in finite element methods, Handbook of numerical analysis, Vol. II, Handb. Numer. Anal., II, North-Holland, Amsterdam, 1991, pp. 353-522. MR 1115238

Universität der Bundeswehr München, 85577 Neubiberg, Germany

E-mail address: Thomas.Apel@unibw.de

Universität der Bundeswehr München, 85577 Neubiberg, Germany

E-mail address: Johannes.Pfefferer@unibw.de

Universität Duisburg-Essen, Fakultät für Mathematik, Thea-Leymann-Str. 9, 45127 Essen, Germany

E-mail address: Arnd.Roesch@uni-due.de 